\title{
Criticality for the Gehring link problem
}

\author{
JASON CANTARELLA \\ JOSEPH H G FU \\ ROB KUSNER \\ JOHN M SULLIVAN \\ NANCY C WRINKLE
}

\begin{abstract}
In 1974, Gehring posed the problem of minimizing the length of two linked curves separated by unit distance. This constraint can be viewed as a measure of thickness for links, and the ratio of length over thickness as the ropelength. In this paper we refine Gehring's problem to deal with links in a fixed link-homotopy class: we prove ropelength minimizers exist and introduce a theory of ropelength criticality.

Our balance criterion is a set of necessary and sufficient conditions for criticality, based on a strengthened, infinite-dimensional version (Theorem 5.4) of the KuhnTucker theorem. We use this to prove that every critical link is $C^{1}$ with finite total curvature. The balance criterion also allows us to explicitly describe critical configurations (and presumed minimizers) for many links including the Borromean rings. We also exhibit a surprising critical configuration for two clasped ropes: near their tips the curvature is unbounded and a small gap appears between the two components. These examples reveal the depth and richness hidden in Gehring's problem and our natural extension.
\end{abstract}

57M25; 49Q10, 53A04

\section{Introduction}

\author{
Suppose that $A$ and $B$ are disjoint linked Jordan curves in $\mathbb{R}^{3}$ \\ which lie at a distance 1 from each other. \\ Show that the length of $A$ is at least $2 \pi$.
}

—Fred Gehring, 1974

Gehring's problem, which appeared in a conference proceedings [7], was soon solved by Marvin Ortel. Because Ortel's elegant solution was never published, we reproduce it here with his permission: Fix any point $a \in A$; the cone on $A$ from $a$ is a disk spanning $A$. Since $A$ and $B$ are linked, $B$ meets this disk at some point $b \in B$, lying 
on a chord of $A$. Because $\operatorname{Dist}(A, b) \geq 1$, projecting $A$ to the unit sphere $S$ around $b$ does not increase its length. The projection is a closed curve joining two antipodal points on $S$, and so has length at least $2 \pi$. (Further proofs and generalizations to linked spheres in higher dimensions were published by Edelstein and Schwarz [11], Osserman [28] and Gage [12;13].)

The unique minimizing configuration for Gehring's problem is a Hopf link consisting of two congruent circles in perpendicular planes, each passing through the other's center. This leads to a natural question: what are the length-minimizing shapes of other link types when the different components stay unit distance apart? This constraint prevents different components from crossing each other, but we cannot expect to fix the link type exactly. Instead, the natural setting for this problem is Milnor's notion of link homotopy: two links are link-homotopic if one can be deformed into the other while keeping different components disjoint. Clearly one link can be deformed into another while keeping all components at unit distance if and only if they are link homotopic.

We will define the link-thickness of a link to be the minimum distance between different components. The problem we consider is then to minimize length in a link-homotopy class, subject to the constraint of fixed link-thickness. Equivalently, we could minimize the link-ropelength of the link, meaning the quotient of length over thickness.

In [4], we found length-minimizing links under a similar constraint: that a normal tube of diameter one around the link stay embedded. It is easy to see that the examples constructed there (like the one in Figure 4) are also global minima (in their respective link-homotopy classes) for the Gehring problem. The focus of this paper will be on critical configurations. Our main result is a balance criterion (Theorem 6.1, Corollary 6.3), which states that a link is link-ropelength critical if and only if the tension force in the curve is balanced by a system of compressive forces between pairs of points on different components of $L$ realizing the minimum distance.

This balance criterion is based mainly on an improved, infinite-dimensional version (Theorem 5.4) of the Kuhn-Tucker theorem on constrained optimization, which is essentially a very general method of Lagrange multipliers. The other key technical element is a careful application of Clarke's differentiation theorem for min-functions (Theorem 3.1).

The direct method shows that there is a (rectifiable) minimizer for link-ropelength in each link-homotopy class. An interesting problem is to determine the regularity of these minimizers or other critical points. The previously known minimizers were $C^{1,1}$ but not $C^{2}$. Our balance criterion allows us to prove that all link-ropelength-critical curves are $C^{1}$ with finite total curvature (Proposition 6.5). 
We next consider generalized links, which may include open components with constrained endpoints, or which avoid fixed obstacles. After extending our balance criterion and existence results to this setting, we analyze the problem of the simple clasp. A clasp consists of two linked arcs whose endpoints are constrained to parallel planes (as in Figure 10). A generalization to clasps of different opening angles provides a model for the strands of rope in a woven cloth or net. The balance criterion lets us construct explicit critical configurations (Theorem 9.5) of these generalized links; we conjecture they are the length-minimizers subject to the constraint that the arcs remain at unit distance from each other. Our critical clasp has a number of surprising features, including a point of infinite curvature and a small gap (at the center of the clasp) between the tubes around the two components. This configuration is $C^{1,2 / 3}$ and may represent the worst regularity of any critical curve.

We end by constructing a ropelength-critical configuration (and presumed minimizer) for the Borromean rings. In all the other known critical configurations for closed links, each component is a convex plane curve built from straight segments and arcs of circles. In our Borromean rings, the components are still planar, but are nonconvex and are built from different pieces including parts of a clasp curve. In a sense, this is the first nontrivial example of a ropelength-critical link.

Our methods will have a number of other applications. In particular, we have used them to describe critical configurations for the "standard" ropelength problem for knots and links: minimize the length of a $C^{1}$ link subject to the constraint that the normal neighborhood of unit diameter remains embedded. We will publish these results in a sequel [2] to the current paper. We can also consider minimization not of length but of other objective functions like elastic bending energy, again subject to a thickness constraint. Analogs of our balance criterion may be useful in describing other flexible mechanisms, such as thick surfaces.

We note that von der Mosel and Schuricht [33] have used a similar approach (via Clarke's theorem and a functional-analytic version of Lagrange multipliers) to derive necessary, but not sufficient, conditions for criticality for the ropelength functional of [4]. We will treat the same functional in our forthcoming sequel [2] and will offer a comparison of the two methods there. We also note that Starostin has given [34] an independent derivation of the tight clasp of Section 9, though he does not prove that it is critical. 


\section{Link-thickness for closed links}

In order to reformulate Gehring's problem, we first establish some basic terminology. Remember that a compact, oriented 1-manifold-with-boundary $M$ is a finite union of components, each of which is homeomorphic to a circle $\mathbb{S}^{1}$ or an interval $[0,1]$.

Definition A parametrized curve is a mapping from a compact, oriented 1-manifoldwith-boundary $M$ to $\mathbb{R}^{3}$. Two parametrized curves are equivalent if they differ by an orientation-preserving reparametrization (thatis, by composition with an orientationpreserving self-homeomorphism of $M$ ). A curve $L$ in $\mathbb{R}^{3}$ is an equivalence class of parametrized curves. We say $L$ is closed when each component of its domain $M$ is a circle, that is, when its boundary $\partial L$ is empty.

Even though our curves may have self-intersections, we will usually refer to points on the curve as if they were simply points of its image in $\mathbb{R}^{3}$. The meaning should be clear from context.

The length Len $(L)$ of any curve $L$ is defined to be the supremal length of all polygons inscribed in $L$. A curve has finite length, or is rectifiable, if and only if it has a Lipschitz (that is, $C^{0,1}$ ) parametrization. One such parametrization is then by arclength $s$. Any rectifiable curve has a well-defined unit tangent vector $T=\mathrm{d} L / \mathrm{d} s$ almost everywhere.

Definition The link-thickness LThi $(L)$ of a curve $L$ is the minimum distance between points on different components of $L$. This is the supremal $\varepsilon$ for which the $(\varepsilon / 2)-$ neighborhoods of the components of $L$ are disjoint.

For now, we will consider only the case of closed curves, where each component is a circle. (We will deal with generalized links—-with endpoint constraints-later in Section 8.) So suppose we start with a closed curve $L$ and we want to minimize length under the constraint that the link-thickness remains at least one. Since we can rescale any link to have LThi $\geq 1$, this problem is the same as minimizing (link-) ropelength, the quotient of length by link-thickness.

The thickness constraint naturally prevents different components from passing through each other, but does not prevent any given component from changing its knot type through self-intersections. This is the setting for Milnor's work on link homotopy:

Definition A link is a closed curve with disjoint components. The link-homotopy class of a link $L$, denoted $[[L]]$, is the set of curves homotopic to $L$ through configurations that keep different components of $L$ disjoint. 
Note that, for our purposes, configurations of $L$ where some components have selfintersections are still considered to be links, and are included in $[[L]]$.

For two-component links, Milnor [25] showed that linking number is the only linkhomotopy invariant. For links of many components, the topological situation is more complicated, but a complete classification of links up to link homotopy was provided by Habegger and Lin [16]. We will prove in Section 6 that in every link-homotopy class there is a curve minimizing ropelength. We show these minimizers are always $C^{1}$, though our examples suggest that they may not always have bounded curvature.

\section{The derivative of link-thickness}

We want to define critical configurations of $L$ subject to the thickness constraint LThi $(L) \geq 1$. Because LThi is defined as the minimum of a collection of distances between points on different components, the equation LThi $\geq 1$ acts like a collection of many constraints. To make this notion precise, we will apply a theorem of Clarke to compute the derivative of LThi as we vary the curve $L$.

Given any curve $L$, let $L^{(2)}$ be the compact set of all unordered pairs $\{x, y\}$ of points on distinct components of $L$. The link-thickness of $L$ is simply the minimum over $L^{(2)}$ of the distance function $\operatorname{Dist}\{x, y\}:=|y-x|$.

We often want to consider a continuous deformation $L_{t}$ of a curve $L$ : fixing any parametrization $f$ of $L$, that means a continuous family $f_{t}$ of parametrized curves with $f_{0}=f$. (When we reparametrize $L$, we apply the same reparametrization to $L_{t}$ at all times $t$.) We assume that $L_{t}$ is $C^{1}$ in $t$; the initial velocity of $L_{t}$ will then be given by some (continuous, $\mathbb{R}^{3}$-valued) vector field $\xi$ along $L$. We let $\operatorname{VF}(L)$ denote the space of all such vector fields. Formally, these are sections of the bundle $f^{*} T \mathbb{R}^{3}$ pulled back from the tangent bundle of $\mathbb{R}^{3}$ by the parametrization $f$ of $L$. Identifying any tangent space to $\mathbb{R}^{3}$ with $\mathbb{R}^{3}$ itself, this is simply a map from the domain $M$ to $\mathbb{R}^{3}$. Again, when we reparametrize a curve $L$, we apply the same reparametrization to any vector field $\xi$.

Consider a curve $L$ with $\operatorname{LThi}(L)>0$. If $L_{t}$ is a continuous deformation of $L$, with initial velocity given by some $\xi \in \operatorname{VF}(L)$, then for each pair $\{x, y\} \in L^{(2)}$, we clearly have

$$
\delta_{\xi} \operatorname{Dist}\{x, y\}:=\left.\frac{\mathrm{d}}{\mathrm{d} t}|y-x|\right|_{t=0}=\frac{\left\langle\xi_{y}-\xi_{x}, y-x\right\rangle}{|y-x|} .
$$

(Even if $L$ is not embedded, the condition LThi $(L)>0$ implies $x$ and $y$ cannot coincide in $\mathbb{R}^{3}$, so this formula is always meaningful.) 
A function like LThi, defined as the minimum of a compact family of smooth functions, is sometimes called a min-function. Clarke's differentiation theorem for min-functions says that - just as in the case when the compact family is finite-the derivative of a min-function is the smallest derivative of those smooth functions that achieve the minimum. More precisely, specializing [6, Theorem 2.1] to the case we need, we have:

Theorem 3.1 (Clarke) Suppose for some compact space $K$ and some $\varepsilon>0$, we have a family of $C^{1}$ functions $f_{k}:(-\varepsilon, \varepsilon) \rightarrow \mathbb{R}$, for $k \in K$. Suppose further that $f_{k}(t)$ and $f_{k}^{\prime}(t)$ are lower semicontinuous on $K \times(-\varepsilon, \varepsilon)$. Let $f(t):=\min _{k \in K} f_{k}(t)$. Then $f$ has one-sided derivatives, and

$$
\left.\frac{\mathrm{d} f}{\mathrm{~d} t^{+}}\right|_{t=0}=\min _{k \in K_{0}} f_{k}^{\prime}(0)
$$

where $K_{0}:=\left\{k \in K: f_{k}(0)=f(0)\right\}$ is the set of $k$ for which the minimum in the definition of $f$ is achieved when $t=0$.

To apply this theorem to thickness, suppose we have a variation $L_{t}$ of the curve $L$, and let $\xi \in \operatorname{VF}(L)$ be its initial velocity. The link-thickness LThi $(L)$ is written as a minimum over $K=L^{(2)}$ of the pairwise distance. Clarke's theorem picks out those pairs achieving the minimum: $K_{0}$ is the set of pairs achieving the minimum distance $\operatorname{LThi}(L)$.

In rigidity theory, the vertices of a tensegrity framework are joined by bars whose length is fixed, cables whose length can shrink but not grow, and struts whose length can grow but not shrink (compare Roth and Whiteley [31]). Thus, we borrow the term "strut" to describe the pairs in $K_{0}$ :

Definition An unordered pair of points $\{x, y\}$ on different components of $L$ is a strut if $|y-x|=\operatorname{LThi}(L)$. The space of all struts of $L$ is denoted $\operatorname{Strut}(L) \subset L^{(2)}$.

Struts correspond to points of contact between tubes around the different components of $L$. Our balance criterion will show how the segment $\overline{x y}$ can be viewed as carrying a force pushing outward on its endpoints.

Applying Clarke's theorem to link-thickness, we get:

Corollary 3.2 For any curve $L$, and any variation vector field $\xi \in \operatorname{VF}(L)$, the (onesided) first variation of link-thickness is

$$
\delta_{\xi}^{+} \operatorname{LThi}(L)=\min _{\operatorname{Strut}(L)} \delta_{\xi} \operatorname{Dist}\{x, y\} .
$$


Note that $\delta_{\xi} \operatorname{Dist}\{x, y\}$ is a continuous function of $x$ and $y$, and for any fixed $\{x, y\}$ is a linear function of the variation $\xi$, being the derivative of a smooth function.

Therefore we can collect these into a linear operator $A_{\mathrm{S}}=\delta$ Dist from $\operatorname{VF}(L)$ to the space $C(\operatorname{Strut}(L))$ of continuous functions on struts, defined by

$$
\left(A_{\mathrm{S}} \xi\right)(\{x, y\}):=\delta_{\xi} \operatorname{Dist}\{x, y\}=\frac{1}{|y-x|}\left\langle\xi_{y}-\xi_{x}, y-x\right\rangle .
$$

Borrowing again from rigidity theory, where the analogous $A$ is called the rigidity matrix, we will call $A_{\mathrm{S}}$ the rigidity operator for link-thickness.

The corollary above can be rephrased to conclude that a variation $\xi$ decreases LThi $(L)$ to first order if and only if $A_{\mathrm{S}} \xi$ takes at least one negative value on $\operatorname{Strut}(L)$.

Note that, while the corollary says that link-thickness has a directional derivative $\delta_{\xi}^{+}$LThi in each direction $\xi$, the operator $\delta_{\xi}^{+}$LThi is not linear in $\xi$. For instance, when one component of a link is between two others, it is easy to have both $\delta_{\xi}^{+}$LThi $<0$ and $\delta_{-\xi}^{+}$LThi $<0$. We write the superscript ${ }^{+}$to emphasize that these are only one-sided derivatives. There is, however, a form of superlinearity:

Corollary 3.3 For any curve $L$ and any $\xi, \eta \in \operatorname{VF}(L)$, we have

$$
\delta_{\xi+\eta}^{+} \operatorname{LThi}(L) \geq \delta_{\xi}^{+} \operatorname{LThi}(L)+\delta_{\eta}^{+} \operatorname{LThi}(L) .
$$

Proof This follows immediately from the linearity of $A_{\mathrm{S}}$ and the general fact that $\min (f+g) \geq \min f+\min g$. We have

$$
\begin{aligned}
\min A_{\mathrm{S}}(\xi+\eta)\{x, y\} & =\min \left(A_{\mathrm{S}} \xi+A_{\mathrm{S}} \eta\right)\{x, y\} \\
& \geq \min A_{\mathrm{S}} \xi\{x, y\}+\min A_{\mathrm{S}} \eta\{x, y\},
\end{aligned}
$$

where the minima are taken over all $\{x, y\} \in \operatorname{Strut}(L)$.

We will be interested in the adjoint $A_{\mathrm{S}}^{*}$ of the rigidity operator, so we first consider the dual function spaces. By the Riesz representation theorem, we know that $C^{*}$ (Strut) is the space of signed Radon measures on the space $\operatorname{Strut}(L)$ of struts. Similarly $\operatorname{VF}^{*}(L)$ is the space of what we will call forces along $L$, namely vector-valued Radon measures on $L$.

The adjoint operator $A_{\mathrm{S}}^{*}$ now associates to any measure $\mu$ on struts a force $A_{\mathrm{S}}^{*} \mu$ along $L$. Geometrically, each pair $\{x, y\}$ acts along the chord $\overline{x y}$, outward at each of 
its endpoints. In formulas,

$$
\begin{aligned}
\int_{L} \xi \mathrm{d} A_{\mathrm{S}}^{*} \mu & =\int_{\text {Strut }} A_{\mathrm{S}} \xi \mathrm{d} \mu \\
& =\int_{x \in L} \int_{y \in L}\left\langle\xi_{y}, \frac{y-x}{|y-x|}\right\rangle \mathrm{d} \mu(x, y),
\end{aligned}
$$

where we have lifted $\mu$ to a symmetric measure $\mu(x, y)$ on ordered pairs. Physically, we think of $\mu$ as giving the strengths of compressive forces within the struts, and $A_{\mathrm{S}}^{*}$ as the operation that integrates these strut forces to give a net force along the curve $L$.

\section{First variation of length and finite total curvature}

The objective functional we consider in this paper is simply the length $\operatorname{Len}(L)$ of a curve. Since our curves might not be smooth, we need to carefully examine the first variation of length.

Let $L$ be a rectifiable curve parametrized by arclength $s$, with unit tangent vector $T$. Suppose $L_{t}$ is a variation of $L$ under which the motion of each point $x \in L$ is smooth in time with initial velocity $\xi_{x}$, and $\xi \in \mathrm{VF}(L)$ is a Lipschitz function of arclength. Then the standard first-variation calculation shows that

$$
\delta_{\xi} \operatorname{Len}(L):=\left.\frac{\mathrm{d}}{\mathrm{d} t} \operatorname{Len}\left(L_{t}\right)\right|_{t=0}=\int_{L}\left\langle T, \xi^{\prime}\right\rangle \mathrm{d} s,
$$

where $\xi^{\prime}=\mathrm{d} \xi / \mathrm{d} s$ is the arclength derivative, defined almost everywhere along $L$.

If $L$ is smooth enough, we can integrate this by parts to get

$$
\delta_{\xi} \operatorname{Len}(L)=-\int_{L}\left\langle T^{\prime}, \xi\right\rangle \mathrm{d} s-\sum_{x \in \partial L}\langle \pm T, \xi\rangle .
$$

(In the boundary term, the sign is chosen to make $\pm T$ point inward at $x$.) In fact, not much smoothness is required: as long as $T$ is a function of bounded variation, we can interpret $T^{\prime}$ as a measure, and the formula holds in a sense we will now explore.

Following Milnor [24], we recall that the total curvature of a polygon is just the sum of its (exterior) turning angles, and we define the total curvature of any curve to be the supremal total curvature over all inscribed polygons. A rectifiable curve $L$ has finite total curvature if and only if the unit tangent vector $T=L^{\prime}(s)$ is a function of bounded variation. Sometimes the space of all such curves is called $W^{1, \mathrm{BV}}$ or $\mathrm{BV}^{1}$, but we will call it FTC. (See Sullivan [36] for a survey of results on FTC curves.) 
If $L \in \mathrm{FTC}$, it follows that at every point of $L$ there are well-defined left and right tangent vectors $T_{ \pm}$; these are equal and opposite except at countably many points, the corners of $L$. (See, for instance, Royden [32, Sect. 5.2].)

If $L$ is FTC, its tangent $T$ has a distributional derivative $\mathcal{K}$ with respect to arclength: a force (an $\mathbb{R}^{3}$-valued Radon measure) along $L$ that we call the curvature force.

The curvature force has an atom (a point mass or Dirac delta) at each corner $x \in L$, with $\mathcal{K}\{x\}=T_{+}(x)+T_{-}(x)$. On a $C^{2}$ arc of $L$, the curvature force is $\mathcal{K}=\mathrm{d} T=\kappa N \mathrm{~d} s$ and this is absolutely continuous with respect to the arclength or Hausdorff measure $\mathrm{d} s=\mathcal{H}^{1}$.

When $L$ has boundary, we choose to include in $\mathcal{K}$ an atom at each endpoint of $L$, with mass 1 and pointing in the inward tangent direction. This means we need no boundary terms in the formula $\delta_{\xi} \operatorname{Len}(L)=-\int_{L}\langle\xi, \mathrm{d} \mathcal{K}\rangle$.

We say that a vector field $\xi$ along $L$ is smooth if $\xi_{s}$ is a smooth function of arclength. (The arclength parametrization of any rectifiable curve is essentially unique, so this makes sense.) The set of all smooth vector fields will be denoted $\mathrm{VF}^{\infty}(L)$.

The first variation $\delta \operatorname{Len}(L)$ can be viewed as a linear functional on smooth vector fields $\xi \in \mathrm{VF}^{\infty}(L)$. As such a distribution, it has order zero, by definition, if $\delta_{\xi} \operatorname{Len}(L)=$ $\int_{L}\left\langle T, \xi^{\prime}\right\rangle \mathrm{d} s$ is bounded by $C \sup _{L}|\xi|$ for some constant $C$. This happens exactly when we can perform the integration by parts.

We collect these results as:

Lemma 4.1 Given any rectifiable curve $L$, the following conditions are equivalent:

(a) $L$ is FTC.

(b) The first variation $\delta \operatorname{Len}(L)$ has distributional order zero.

(c) There exists a curvature force measure $\mathcal{K}$ along $L$ such that $\delta_{\xi} \operatorname{Len}(L)=$ $-\int_{L}\langle\xi, \mathrm{d} \mathcal{K}\rangle$.

An FTC curve $L$ is $C^{1}$ exactly when it has no corners, that is, when $\mathcal{K}$ has no atoms (except at the endpoints). It is furthermore $C^{1,1}$ when $T$ is Lipschitz, or equivalently when $\mathcal{K}$ is absolutely continuous (with respect to arclength) and has bounded RadonNikodym derivative $\mathrm{d} \mathcal{K} / \mathrm{d} \mathcal{H}^{1}=\kappa N$. In previous work on ropelength (for example $[4 ; 14])$, the thickness measure had an upper bound for curvature built in, meaning that any curve of positive thickness was automatically $C^{1,1}$. This is not true for the link-thickness, so we do not expect the same regularity results to hold here. 


\section{Constrained criticality and the Kuhn-Tucker theorem}

We will review constrained minimization problems in a finite setting, before generalizing to the setting we will need for our ropelength problems. Suppose we want to minimize a $C^{1}$ function $f: \mathbb{R}^{n} \rightarrow \mathbb{R}$ inside the admissible region defined by a finite collection of $C^{1}$ inequality constraints $g_{i} \geq 0$. A constraint $g_{i}$ is active at $p \in \mathbb{R}^{n}$ if $g_{i}(p)=0$.

Definition We say that $p$ is a constrained critical point for minimizing $f$ if, for any tangent vector $v$ at $p$ with $D_{v} f<0$, we have $D_{v} g_{i}<0$ for some active $g_{i}$. That is, $p$ is critical if there is no direction $v \in \mathbb{R}^{n}$ that reduces $f$ to first order while preserving all constraints to first order.

Note that the criticality conditions for minimizing $f$ and $-f$ are quite different; in particular local maxima for $f$ are rarely critical points for minimizing $f$, while local minima for $f$ usually - though not always — are.

Example 5.1 Suppose we minimize $f(x, z):=x$ on the halfplane $x \geq 0$ in $\mathbb{R}^{2}$, subject to

$$
g_{1}:=\left(x^{2}-1\right)^{3}-z \geq 0, \quad g_{2}:=z \geq 0 .
$$

The admissible region has an outward-pointing cusp, shown in Figure 1. The tip of this cusp, at $p=(1,0)$, is the global minimum of $f$ over the admissible region, but it is not critical: the directional derivatives in the direction $v=(-1,0)$ are $D_{v} f=-1$ but $D_{v} g_{i}=0$.

To deduce that a local minimum of $f$ is critical according to our definition, an additional regularity hypothesis will be required. However, critical points can be exactly characterized by a Lagrange multiplier theorem (compare [17]):

Theorem 5.2 (Modified Kuhn-Tucker Theorem) A point $p$ is constrained-critical for minimizing $f$ if and only if the gradient $\nabla f$ is a positive linear combination of the gradients $\nabla g_{j}$ of the constraints $g_{j}$ active at $p$.

The geometric intuition behind this theorem is easy to understand: Only the active constraints matter, and being inequality constraints they can only act positively. If there were some component of $-\nabla f$ not canceled by the $\nabla g_{j}$, that would give an admissible direction to move which decreases $f$.

Unlike in the classical Kuhn-Tucker theorem, we do not need additional regularity hypotheses on the point $p$, which may surprise those familiar with optimization theory. The explanation is that we are interested in critical points, while the classical theorem 


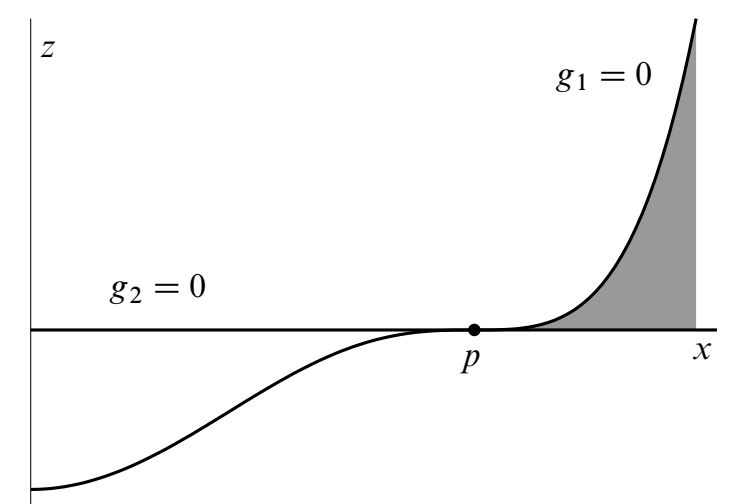

Figure 1: In this illustration of Example 5.1, the admissible region for the two constraints $g_{1}=\left(x^{2}-1\right)^{3}-z \geq 0$ and $g_{2}=z \geq 0$ is shaded. The MangasarianFromovitz constraint qualification fails at the cusp point $p=(1,0)$ because $\nabla g_{1}$ and $\nabla g_{2}$ are equal and opposite there.

deals with minima of $f$. And as we saw above, not every minimum of $f$ is critical. But just as in the classical theory, criticality will be guaranteed if we add the hypothesis that the Mangasarian-Fromovitz constraint qualification [20] holds for a local minimum.

Definition A point $p$ is constraint-qualified (in the sense of Mangasarian and Fromovitz) if there is a direction $v$ such that for all constraints $g_{j}$ active at $p$ we have $D_{v} g_{j}>0$.

We note that this condition fails at the point $p=(1,0)$ in Example 5.1 above, which was minimal but not critical.

Proposition 5.3 If $p$ is a local minimum for $f$ when constrained by $\left\{g_{i} \geq 0\right\}$, and $p$ is constraint-qualified, then $p$ is constrained-critical for minimizing $f$.

We have omitted proofs of the theorem and proposition above because they are standard and are also special cases of our infinite-dimensional generalizations below.

\subsection{A generalized Kuhn-Tucker theorem}

Note that in Theorem 5.2, the functions $f$ and $g_{i}$ might as well be replaced by linear functions-their differentials at $p$. We view this as the linear-algebraic core of the Kuhn-Tucker theorem.

We will now derive an infinite-dimensional version, where the linear functional $f$ is defined on an arbitrary vector space $X$, and the finite family of constraints $g_{i}$ is replaced by a family $A_{y}$, where $y$ ranges over some compact space $Y$. 
While our theorem does not mention optimization directly, it will be the engine that drives all of the optimization theorems of this paper.

As usual, we let $C(Y)$ be the Banach space of continuous functions on $Y$ with the sup norm $\|\cdot\|$, and let $P \subset C(Y)$ be the closed positive orthant consisting of nonnegative functions. Then the dual space $C^{*}(Y)$ consists of all signed Radon measures on $Y$, and $P^{*} \subset C^{*}(Y)$ is the cone of positive measures.

Note that any function $z \in C(Y)$ can be decomposed into positive and negative parts: $z=z^{+}-z^{-}$with $z^{ \pm} \in P$. Then we have $\left\|z^{-}\right\|=\operatorname{Dist}(z, P)$.

Theorem 5.4 Let $X$ be any vector space and $Y$ be a compact topological space. For any linear functional $f$ on $X$ and any linear map $A: X \rightarrow C(Y)$, the following are equivalent:

(a) There exists $\varepsilon>0$ such that $\left\|(A \xi)^{-}\right\| \geq \varepsilon$ for all $\xi \in X$ with $f(\xi)=-1$.

(b) There exists a positive Radon measure $\mu \in P^{*}$ such that $f(\xi)=\mu(A \xi)$ for all $\xi \in X$.

This theorem is comparable to the generalized Kuhn-Tucker theorem of Luenberger [19, page 249]. His theorem, restated to apply to the linear Gateaux differentials ( $f$ and $A$ ) of the original objective and constraint functions on $X$, says:

Theorem 5.5 (Luenberger) Let $X$ and $Z$ be vector spaces, with a norm given on $Z$, and let $P \subset Z$ be a closed convex cone with nonempty interior. Let $f: X \rightarrow \mathbb{R}$ be a linear functional and $A: X \rightarrow Z$ be a linear map. Assume that whenever $A \xi \in P$ we have $f(\xi) \geq 0$, and that $A \xi$ lies in the interior of $P$ for some $\xi \in X$. Then there exists $\mu \in P^{*}$ such that $f(\xi)=\mu(A \xi)$ for all $\xi \in X$.

While our version applies only to the case $Z=C(Y)$, our hypotheses (a) on $f$ and $A$ are somewhat weaker than those imposed by Luenberger-they are necessary as well as sufficient for (b) the existence of $\mu$.

To understand our overall strategy, consider the linear map $(f, A): X \rightarrow \mathbb{R} \times C(Y)$. As we will see below, (a) implies that the image of $(f, A)$ avoids the interior of the orthant $\mathbb{R}^{-} \times P$.

To gain some intuition, let us specialize to the case where $X=\mathbb{R}^{m}$. We can rephrase (b) to say that some vector in the kernel of the adjoint map $(f, A)^{*}$ is in $\mathbb{R}^{-} \times P$. When $Y$ has finite cardinality $n$, we put the standard Euclidean inner product on $\mathbb{R} \times C(Y) \cong \mathbb{R}^{n+1}$, and identify this space with its dual. Then the kernel of $(f, A)^{*}$ and the image of $(f, A)$ are orthogonal complements in $\mathbb{R}^{n+1}$. The standard Farkas 
alternative (see Figure 2) says that, given any closed orthant in $\mathbb{R}^{n+1}$, it must intersect one out of any pair of orthogonal complements. Our argument in the general case, when $Y$ might be infinite and $X$ infinite dimensional, will be guided by this intuition.
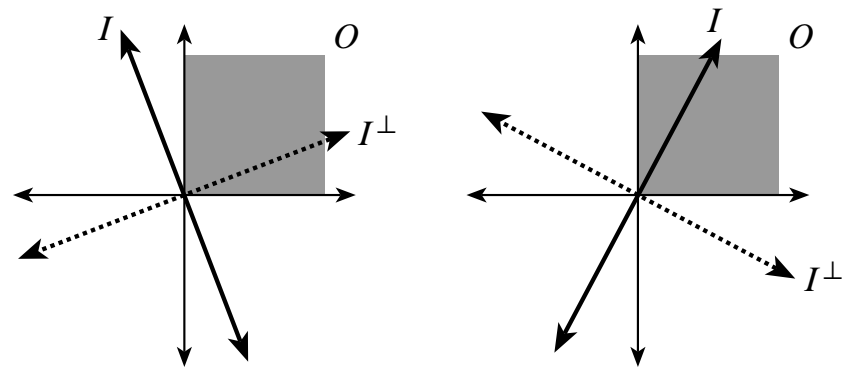

Figure 2: One version of the Farkas alternative states that, given any closed orthant $O$ in a inner product space, it must intersect at least one out of any pair of orthogonal complements $I$ and $I^{\perp}$.

Proof of Theorem 5.4 One direction is easy: suppose we have a positive Radon measure $\mu$ so that for each $\xi \in X$,

$$
f(\xi)=\int_{Y} A \xi \mathrm{d} \mu
$$

For any $\xi \in X$ with $f(\xi)=-1$, write $z:=A \xi \in C(Y)$. We have $\int z \mathrm{~d} \mu=-1$, and since $\mu$ is a positive measure, we can replace the function $z$ with its negative part to conclude that $\int z^{-} \mathrm{d} \mu \geq 1$. Furthermore $\mu$ has finite mass mass $(\mu):=\int \mathrm{d} \mu<\infty$ by the Riesz theorem. Therefore

$$
\operatorname{Dist}(z, P)=\left\|z^{-}\right\| \geq 1 / \operatorname{mass}(\mu)>0 .
$$

This completes the proof that (b) implies (a).

To prove the converse, first give $\mathbb{R} \times C(Y)$ the Euclidean combination of the sup norms on $\mathbb{R}$ and $C(Y)$ :

$$
\|(a, g)\|=\sqrt{a^{2}+\|g\|^{2}} .
$$

Now consider the orthant $O:=[-1, \infty) \times P$. Our hypothesis (a) implies that there is positive distance between $O$ and the image $I:=(f, A)(X)$ of the linear map $(f, A)$. Take sequences $\left(f\left(\xi_{i}\right), A \xi_{i}\right)$ in $I$ and $\left(t_{i}, z_{i}\right)$ in $O$, whose pairwise distance approaches $\operatorname{Dist}(I, O)$. That is, setting

$$
v_{i}:=\left(t_{i}-f\left(\xi_{i}\right), z_{i}-A \xi_{i}\right)
$$

we have $\left\|v_{i}\right\| \rightarrow \operatorname{Dist}(I, O)$. 
We first claim that we can assume that $v_{i} \in \mathbb{R}^{-} \times P$. Certainly we can assume the equality $z_{i}=\left(A \xi_{i}\right)^{+}$, since this positive part of the function $A \xi_{i}$ realizes the distance $\left\|z_{i}-A \xi_{i}\right\|=\left\|\left(A \xi_{i}\right)^{-}\right\|=\operatorname{Dist}\left(A \xi_{i}, P\right)$. Then $z_{i}-A \xi_{i}=-\left(A \xi_{i}\right)^{-} \in P$. Similarly, $\operatorname{Dist}\left(f\left(\xi_{i}\right),[-1, \infty)\right)$ is $-1-f\left(\xi_{i}\right)$ if this is positive (and is zero otherwise), so we may assume $t_{i}=\min \left(-1, f\left(\xi_{i}\right)\right)$. Thus $t_{i} \leq f\left(\xi_{i}\right)$, so $t_{i}-f\left(\xi_{i}\right) \leq 0$. This proves the first claim.

We now have a geometric problem: from Figure 2 we see there is a special case where both the image of $(f, A)$ and its orthogonal complement lie on the boundary of $\mathbb{R}^{-} \times P$. If this happens, then the closure $\bar{I}$ intersects either the subspace $\mathbb{R} \times\{0\}$ or $\{0\} \times P$. The second case does not trouble us, but the first would cause us problems later; we now show that our assumption (a) rules it out. To do so, we think about the setup above geometrically: if $\bar{I}$ intersects $\mathbb{R} \times\{0\}$, then we expect that $t_{i}-f\left(\xi_{i}\right) \rightarrow 0$.

Thus our second claim is that we can assume the $t_{i}-f\left(\xi_{i}\right)$ are uniformly negative. If not, $\lim f\left(\xi_{i}\right) \leq-1$, so without loss of generality, we can rescale $\xi_{i}$ down so that $f\left(\xi_{i}\right)=-1$. That means $\left(-1, A \xi_{i}\right) \in I$. By hypothesis (a) we know

$$
d_{i}:=\operatorname{Dist}\left(\left(-1, A \xi_{i}\right), O\right)=\operatorname{Dist}\left(A \xi_{i}, P\right) \geq \varepsilon
$$

for some fixed $\varepsilon>0$. Since we are using the Euclidean combination of the norms on $\mathbb{R}$ and $C(Y)$, the distance from any rescaling by $k$ of $\left(-1, A \xi_{i}\right)$ to $O$ is given by the Euclidean distance from $\left(-k, k\left\|A \xi_{i}^{-}\right\|\right)$to $(-1,0)$. And we can use plane geometry to see that rescaling by $1-\varepsilon^{2}$ brings us closer to $O$ :

$$
\operatorname{Dist}\left(\left(1-\varepsilon^{2}\right)\left(-1, A \xi_{i}\right), O\right)=d_{i} \sqrt{1-2 \varepsilon^{2}+\varepsilon^{4}\left(1+1 / d_{i}^{2}\right)} \leq d_{i} \sqrt{1-\varepsilon^{2}+\varepsilon^{4}} .
$$

We can always assume that $\varepsilon<1$, so the constant $\sqrt{1-\varepsilon^{2}+\varepsilon^{4}}$ is less than 1 . Therefore

$$
\begin{aligned}
\operatorname{Dist}(I, O) & \leq \lim \operatorname{Dist}\left(\left(1-\varepsilon^{2}\right)\left(-1, A \xi_{i}\right), O\right) \\
& <\lim \operatorname{Dist}\left(\left(-1, A \xi_{i}\right), O\right) \\
& =\operatorname{Dist}(I, O) .
\end{aligned}
$$

This contradiction proves the second claim.

We have proved that the $v_{i}$ are in $\mathbb{R}^{-} \times P$. Using the Hahn-Banach theorem, for each $i$ we can find a linear functional $\left(c_{i}, v_{i}\right) \in \mathbb{R} \times C^{*}(Y)$ that vanishes on $I$, satisfies $\left(c_{i}, v_{i}\right)\left(v_{i}\right)=1$, and has norm

$$
\left\|\left(c_{i}, v_{i}\right)\right\|=1 / \operatorname{Dist}\left(v_{i}+I,(0,0)\right) .
$$

Because the $\mathbb{R}$-components of $v_{i}$ are uniformly negative, so are the $c_{i}$. 
Using Alaoglu's theorem, the $\left(c_{i}, v_{i}\right)$ have a subsequence converging in the weak* topology to a limit functional $(c, v)$; we have $c<0$ and its norm is bounded above by $1 / \lim \operatorname{Dist}\left(v_{i},-I\right)=1 / \operatorname{Dist}(I, O)$.

Setting $\mu:=v /|c| \in C^{*}(Y)$, we claim this will be the Radon measure in statement (b). By construction, $(-1, \mu)$ vanishes on $I$, meaning that for $\xi \in X$, we have

$$
-f(\xi)+\int_{Y} A \xi \mathrm{d} \mu=0 .
$$

(Notice that we have used the additional geometric information that $I$ does not approach $\mathbb{R} \times\{0\}$ in an essential way; if it did, then $c$ would vanish, and we could not rescale $v$ by $1 /|c|$ to obtain the equation above.)

It remains only to show that $\mu$ is positive. In an inner product space, this would be obvious: each $v_{i}$ would be positive (since it was dual to a positive $z_{i}-A \xi_{i}$ ), and $v$ would be a limit of positive measures. But our $v_{i}$ were constructed implicitly by the Hahn-Banach theorem, and so might include negative pieces. We now address this problem.

We can decompose each $v_{i}$ into its positive and negative parts $v_{i}=v_{i}^{+}-v_{i}^{-}$, with $\operatorname{mass}\left(v_{i}\right)=\operatorname{mass}\left(v_{i}^{+}\right)+\operatorname{mass}\left(v_{i}^{-}\right)$. In order to show $v$ is positive, we will prove that $\lim \operatorname{mass}\left(v_{i}^{+}\right)=\lim \operatorname{mass}\left(v_{i}\right)$. By construction, we know that

$$
1=\left(c_{i}, v_{i}\right)\left(v_{i}\right)=c_{i}\left(t_{i}-f\left(\xi_{i}\right)\right)+\int_{Y} z_{i}-A \xi_{i} \mathrm{~d} v_{i} .
$$

Since $z_{i}-A \xi_{i} \in P$, we have

$$
\int_{Y} z_{i}-A \xi_{i} d v_{i} \leq \int_{Y} z_{i}-A \xi_{i} d v_{i}^{+} \leq\left\|z_{i}-A \xi_{i}\right\| \operatorname{mass}\left(v_{i}^{+}\right) .
$$

Using Cauchy-Schwarz, and the two equations above, we get

$$
1 \leq\left\|v_{i}\right\| \sqrt{\left|c_{i}\right|^{2}+\left(\operatorname{mass}\left(v_{i}^{+}\right)\right)^{2}} .
$$

Now $\left\|v_{i}\right\|$ converges to $\operatorname{Dist}(I, O)$, so we find $\lim \left\|\left(c_{i}, v_{i}^{+}\right)\right\| \geq 1 / \operatorname{Dist}(I, O)$. But the limit of $\left\|\left(c_{i}, v_{i}\right)\right\|$ (which cannot be smaller) equals $1 / \operatorname{Dist}(I, O)$. Therefore, $\lim \operatorname{mass}\left(v_{i}^{+}\right)=\lim \operatorname{mass}\left(v_{i}\right)$, completing the proof.

To apply Theorem 5.4 to optimization problems, we will let $X$ be the space of variations $\xi$ of our given configuration and $Y$ be the set of active constraints. Then we let $f(\xi)$ and $A \xi(y)$ be the directional derivatives of the objective function and of the constraint $y \in Y$. 
In this context, a configuration satisfying condition (a) of Theorem 5.4 is called strongly critical, and one satisfying (b) is balanced. The theorem then says that a configuration is strongly critical if and only if it is balanced.

Note that our strong criticality is indeed stronger than a simple criticality condition, which would say that whenever $f(\xi)=-1$ we have $\operatorname{Dist}(A \xi, P)>0$, or equivalently that no $\xi$ has $f(\xi)<0$ but $A \xi \in P$.

Example 5.6 With $X=\mathbb{R}^{2}$ and $Y=[0,1]$, we can set $f\left(x_{1}, x_{2}\right)=x_{1}$ and

$$
A\left(x_{1}, x_{2}\right)(y)=2 x_{1} \sqrt{y-y^{2}}+x_{2} y
$$

to give an example that is critical, but not strongly critical (and thus not balanced).

However, when $Y$ is a finite set (with the discrete topology), strong criticality is equivalent to criticality. For suppose whenever $f(\xi)=-1$ we have $\operatorname{Dist}(A \xi, P)>0$, but there is no uniform lower bound $\varepsilon>0$ on this distance. For each $y \in Y$, we know that $A \xi(y)$ is a linear functional on $\xi$. Since there are only finitely many $y$, the graph of $\min _{y \in Y} A \xi(y)$ describes a polyhedron in $X \times \mathbb{R}$. Since the supremum over $\xi \in X$ is finite (we know it is nonpositive), it is achieved (at some $\xi$ corresponding to a vertex of this polyhedron). But for any $\xi$, the value is negative, so this supremum must be negative.

This allows us to recover the finite-dimensional Kuhn-Tucker theorem: let $X$ be the tangent space to $\mathbb{R}^{n}$ at $p$, let $Y$ be the finite set of active constraints at $p$, and let $f$ and $A$ be the directional derivatives of the objective function and the active constraints. Because $Y$ is finite, (a) is equivalent to the definition of constrained criticality above, and we obtain Theorem 5.2.

\section{The balance criterion for the Gehring problem}

We now have all the tools we need to develop a balance criterion characterizing critical configurations for the link-ropelength problem. We start with definitions of criticality, guided by our version of Kuhn-Tucker.

Definition Suppose $L$ is a rectifiable link with LThi $(L)=\tau$, and consider the Gehring problem of minimizing length subject to the constraint LThi $\geq \tau$. We say that $L$ is:

- a local minimum for link-ropelength if for all $L^{\prime}$ sufficiently $C^{0}$-close to $L$ with $\operatorname{LThi}\left(L^{\prime}\right) \geq \tau$ we have $\operatorname{Len}\left(L^{\prime}\right) \geq \operatorname{Len}(L)$.

- critical if for all $\xi \in \mathrm{VF}^{\infty}(L)$ with $\delta_{\xi} \operatorname{Len}(L)<0$ we have $\delta_{\xi}^{+} \operatorname{LThi}(L)<0$. 
- strongly critical if there exists some $\varepsilon>0$ such that for all $\xi \in \mathrm{VF}^{\infty}(L)$ with $\delta_{\xi} \operatorname{Len}(L)=-1$, we have $\delta_{\xi}^{+} \operatorname{LThi}(L) \leq-\varepsilon$.

With these definitions, we can now apply our Kuhn-Tucker theorem to the Gehring problem.

Theorem 6.1 (Balance Criterion) A link $L$ is strongly critical for length when constrained by link-thickness if and only if there exists a positive Radon measure $\mu$ on $\operatorname{Strut}(L)$ such that, for every smooth vector field $\xi$ along $L$, we have

$$
\delta_{\xi} \operatorname{Len}(L)=\int_{\operatorname{Strut}(L)} A_{\mathrm{S}} \xi \mathrm{d} \mu
$$

where $A_{\mathrm{S}}=\delta$ Dist is the rigidity operator.

Proof We will apply Theorem 5.4 with $X:=\mathrm{VF}^{\infty}(L)$ and $Y:=\operatorname{Strut}(L)$, letting $f:=\delta \operatorname{Len}(L)$ be the derivative of length and $A:=A_{\mathrm{S}}$ be the rigidity operator. We have

$$
\left\|\left(A_{\mathrm{S}} \xi\right)^{-}\right\|=-\min _{\text {Strut }} \delta_{\xi} \operatorname{Dist}\{x, y\}
$$

(when this is nonnegative). By Corollary 3.2, the right-hand side is $-\delta_{\xi}^{+} \operatorname{LThi}(L)$, so that condition (a) from Theorem 5.4 is exactly strong criticality.

\subsection{Smoothness of critical curves}

It is unclear, a priori, how much regularity one should expect for ropelength-critical curves in the Gehring problem. But we can use the balance criterion to deduce immediately that they must have finite total curvature.

Corollary 6.2 If a link $L$ is strongly critical for the Gehring problem, then $L$ is FTC.

Proof The theorem tells us that $L$ can be balanced:

$$
\delta_{\xi} \operatorname{Len}(L)=\int_{\operatorname{Strut}(L)} A_{\mathrm{S}} \xi \mathrm{d} \mu .
$$

But the right-hand side is a distribution of order zero on $\xi$, since

$$
\int_{\operatorname{Strut}(L)} A_{\mathrm{S}} \xi \mathrm{d} \mu \leq \operatorname{mass}(\mu) \sup _{L}|\xi| .
$$

Therefore, by Lemma 4.1, $L \in$ FTC. 
We can now rewrite the conclusion of our balance criterion in terms of the curvature force $\mathcal{K}$ on $L$ and the adjoint $A_{\mathrm{S}}^{*}$ of the rigidity operator.

Corollary 6.3 A link $L$ is strongly critical for link-ropelength if and only if it has finite total curvature and there exists a positive Radon measure $\mu$ on $\operatorname{Strut}(L)$ such that

$$
A_{\mathrm{S}}^{*}(\mu)=-\mathcal{K}
$$

as forces along $L$.

Proof The theorem guarantees that for all smooth $\xi$, we have

$$
\delta_{\xi} \operatorname{Len}(L)=\int_{\operatorname{Strut}(L)} A_{\mathrm{S}} \xi \mathrm{d} \mu
$$

By the corollary, $L$ is FTC, so the left-hand side can be rewritten as $-\int_{L}\langle\xi, \mathrm{d} \mathcal{K}\rangle$. Approximating any continuous vector field uniformly by smooth ones, we find that

$$
-\int_{L}\langle\xi, \mathrm{d} \mathcal{K}\rangle=\int_{\operatorname{Strut}(L)} A_{\mathrm{S}} \xi \mathrm{d} \mu
$$

for all $\xi \in \operatorname{VF}(L)$, or in other words, $-\mathcal{K}=A_{\mathrm{S}}^{*}(\mu)$.

We get an immediate and useful geometric corollary to this balance criterion.

Corollary 6.4 Suppose $L$ is critical for link-ropelength, and $E \subset L$ is a subset with nonzero net (vector) curvature $0 \neq \mathcal{K}(E) \in \mathbb{R}^{3}$. Then there must be at least one strut $\{e, x\}$ with $e \in E$ and $x \notin E$, and $\mathcal{K}(E)$ is in the convex cone generated by the directions $x-e$ of all such struts.

Proof First note that struts from $E$ to $E$ contribute no net force. By the balance criterion, we have $\mathcal{K}(E)=-A_{\mathrm{S}}^{*} \mu(E)$, and the latter is a (positive) weighted sum of vectors $x-e$.

We note that this corollary is the analogue for link-ropelength of von der Mosel and Schuricht's "Characterization of Ideal Knots" [33, Theorem 1].

We next find that critical links are $C^{1}$ as well as FTC:

Proposition 6.5 If $L$ is strongly critical for link-ropelength, then $L$ is $C^{1}$. 
Proof We already know that $L$ has finite total curvature; it is $C^{1}$ precisely when it has no corners, that is, when the curvature force $\mathcal{K}$ has no atoms. If $T_{ \pm}$are the right and left tangent vectors to $L$ at $x$, then $\mathcal{K}(\{x\})=T_{+}+T_{-}$. When $\mathcal{K}(\{x\}) \neq 0$, Corollary 6.4 says there is at least one strut $\{x, y\}$ with $\langle y-x, \mathcal{K}(\{x\})\rangle>0$. That is,

$$
\left\langle y-x, T_{+}\right\rangle+\left\langle y-x, T_{-}\right\rangle>0,
$$

so we must have $\left\langle y-x, T_{+}\right\rangle>0$ or $\left\langle y-x, T_{-}\right\rangle>0$. (See Figure 3.) In either case it follows that there exist points on $L$ near $x$ that are closer to $y$ than $x$ is, which contradicts the hypothesis that $\{x, y\}$ was a strut. This completes the proof.

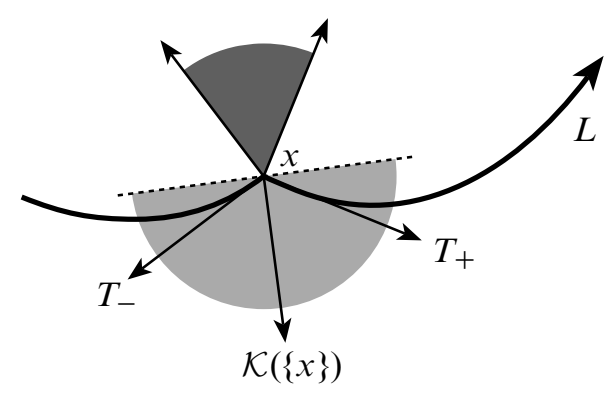

Figure 3: This curve $L$ has a corner at $x$ with left and right tangent vectors $T_{-}$and $T_{+}$, whose sum is the curvature force $\mathcal{K}(\{x\})$ there. If $L$ is to balance, there must be a strut $\{x, y\}$ with $y$ in the open hemisphere (shown in light gray) of vectors with positive inner product with $\mathcal{K}(\{x\})$. But for any $y$ outside the normal cone (shown in dark gray), there are points near $x$ on $L$ that are closer to $y$ than $x$ is. Thus our $\{x, y\}$ cannot be a local minimum of the self-distance function. This contradiction proves that a critical curve cannot have a corner.

The example of the tight clasp in Section 9 shows that critical links need not be $C^{1,1}$ their curvature need not be bounded-but so far this is the worst behavior we can display. We conjecture that the curvature measure is always absolutely continuous with respect to arclength.

\subsection{Constraint qualification in the sense of Mangasarian-Fromovitz}

Corollary 6.3 will be the basic model for balance criteria for generalized links, and for links constrained by other thickness functionals [2]. In some cases, including the link-ropelength for closed links we are treating now, we can improve on this form of the criterion by replacing strong criticality with criticality. This is our next goal. 
In Section 5, we defined a regular or constraint-qualified point for a finite set of constraints $g_{1}, \ldots, g_{n}$ : such a point has some variation direction $v$ such that $D_{v} g_{i}>0$ for all the active $g_{i}$. By Corollary 3.2, the corresponding idea for a link $L$ in the Gehring problem is the existence of a vector field $\xi$ for which $\delta_{\xi}^{+} \operatorname{LThi}(L)>0$. But this is automatic: dilating $L$ increases LThi to first order.

This regularity for our problem allows us to prove that local minima are critical and that critical points are strongly critical.

Proposition 6.6 A link $L$ is critical for the link-ropelength problem if and only if it is strongly critical. If $L$ is a local minimum, then $L$ is critical.

Proof Suppose $L$ is a local minimum but not critical. Then for some $\xi \in \mathrm{VF}^{\infty}(L)$ we have $\delta_{\xi} \operatorname{Len}(L)<0$ but $\delta_{\xi}^{+} \operatorname{LThi}(L) \geq 0$. Then for small enough $t>0$, the link $L+t \xi$ has less length than $L$. This contradicts minimality unless $\delta_{\xi} \operatorname{LThi}(L)=0$ and thickness has decreased (but not to first order). But in this case, we can instead use the rescaled deformation (LThi $(L+t \xi))^{-1}(L+t \xi)$, for which LThi $\equiv 1$. For small $t>0$ these again have less length than $L$, contradicting minimality.

Strong criticality always implies criticality. Conversely, suppose a closed link $L$ is critical but not strongly critical. Then there exists a sequence $\xi_{i} \in \operatorname{VF}(L)$ with $\delta_{\xi_{i}} \operatorname{Len}(L)=-1$ and $\delta_{\xi_{i}}^{+} \operatorname{LThi}(L) \rightarrow 0$. Let $\eta$ be the vector field along $L$ induced by dilation, scaled so that $\delta_{\eta} \operatorname{Len}(L)<1$. Then we observe that $\delta_{\eta+\xi_{i}} \operatorname{Len}(L)<0$ for all $i$. The superlinearity of Corollary 3.3 shows that

$$
\lim \delta_{\eta+\xi_{i}}^{+} \text {LThi } \geq \delta_{\eta}^{+} \text {LThi }>0 .
$$

But then for some $i$, we must have

$$
\delta_{\eta+\xi_{i}} \operatorname{Len}(L)<0 \quad \text { and } \quad \delta_{\eta+\xi_{i}}^{+} \operatorname{LThi}(L)>0,
$$

contradicting the criticality of $L$.

Thus for closed links, a minimizer (or more generally any critical point) for the linkropelength problem is strongly critical, and hence by Corollary 6.3 its curvature force is balanced by some strut force $A_{\mathrm{S}}^{*} \mu$. However, in our generalized ropelength problems, with endpoint constraints and obstacles, constraint qualification will not always hold. Then we will have to be careful about the distinction between criticality and strong criticality. 


\subsection{Existence of minimizers}

We now show that each link-homotopy class contains a globally length-minimizing curve with LThi $\geq 1$.

Proposition 6.7 In a given link-homotopy class $[[L]]$, among all curves with linkthickness at least 1 , there is some $L_{0}$ of minimum length.

Proof We may rescale the initial $L$ so that $\operatorname{LThi}(L) \geq 2$. Thus if the $C^{0}$ distance between $L$ and a link $L^{\prime}$ is less than $1 / 2$ then the straight-line homotopy between them is a link homotopy, and $\operatorname{LThi}\left(L^{\prime}\right) \geq 1$. Taking $L^{\prime}$ to be a standard smoothing of $L$ (for instance, its convolution with a smooth bump function), it follows that $[[L]]$ contains a $C^{\infty}$ link.

In particular, the set of rectifiable links in $[[L]]$ with link-thickness at least 1 is nonempty. Let $L_{1}, L_{2}, \ldots$ be a sequence of such links with lengths tending to the infimal length $\ell$ in this class. By the Arzela-Ascoli theorem, taking a subsequence we may assume that the $L_{i}$ converge in $C^{0}$ to a limit $L_{0}$. Since LThi is continuous with respect to the $C^{0}$ topology, and length is lower semicontinuous, it follows that $\operatorname{LThi}\left(L_{0}\right) \geq 1$ and $\operatorname{Len}\left(L_{0}\right) \leq \ell$. By the remarks of the last paragraph, $L_{0}$ is link homotopic to $L_{i}$ for large $i$, and therefore $L_{0} \in[[L]]$. Thus $\operatorname{Len}\left(L_{0}\right)=\ell$ and $L_{0}$ is the required minimizer.

Since $C^{\infty}$ links are tame, the argument above also shows the following:

Proposition 6.8 There are no wild link-homotopy types with finitely many components.

(This was originally observed by Milnor [25].) Thus in the work to come, we need only to consider tame links.

\section{Examples of critical links}

\subsection{The known length-minimizing links}

In [4], we showed that if one component of a link is linked to $k$ others then its length is at least a certain constant $P_{k}$. Although our theorem was written for the original ropelength problem, the proof is valid for the Gehring problem as well. Whenever a link can be realized with each component having length $P_{k}$, that configuration is thus a 
length-minimizer not only when constrained by thickness but also when constrained by link-thickness. (These are still the only examples known to be ropelength-minimizers.)

To any link $L$ we can associate a graph: the vertices are the components of $L$, and the edges record which pairs are nontrivially linked. For any tree $T$ with $n$ edges, there is a unique link $H(T)$ that is a connect sum of $n$ Hopf links and whose associated graph is $T$.

For many trees $T$ with vertices of sufficiently low degree, we can realize $[[H(T)]]$ explicitly with each component having exactly its minimum possible length $P_{k}$. Even some slightly more complicated links, like the example in Figure 4, whose graph is not a tree, can be realized in this way. The distance between any two linked components

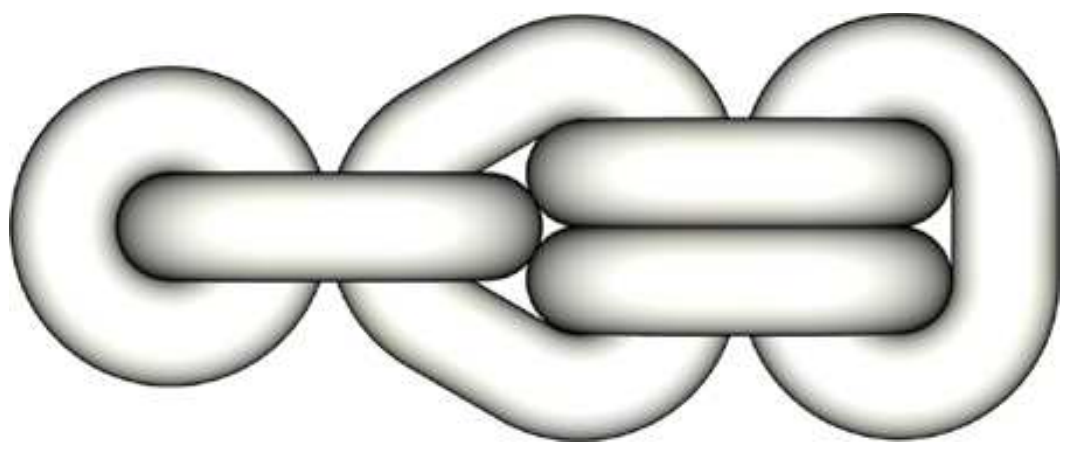

Figure 4: This link of six components is a global minimizer for the linkropelength problem. Each component is a convex plane curve that minimizes its length given the number of other components it links.

is exactly 1. Each component in one of these minimizers is a convex plane curve built from circular arcs of radius 1 and straight segments. It is an outer parallel (at distance $1 / 2$ ) to a shortest curve surrounding $n$ disjoint unit-diameter disks in the plane. (See Figure 5.)

Consider the $n$-star $T_{n}$, the tree with a central vertex incident to all $n$ edges. For $n \leq 5$, the construction above produces a link-ropelength-critical configuration of $H\left(T_{n}\right)$ that is known to be minimizing. We will examine the case $n=2$ in detail, in light of our balance criterion, and then indicate how to produce link-ropelength-critical configurations for all $n$.

Example 7.1 The link $H\left(T_{2}\right)$ is the simple chain of three components, shown in Figure 6. In the ropelength minimizer, the two end components are circles $C_{1}$ and $C_{2}$, while the middle component is a stadium curve $S$. The centers of the circular arcs in $S$ are points $c_{i} \in C_{i}$, while the center of each $C_{i}$ is a point $s_{i} \in S$. The struts are exactly 

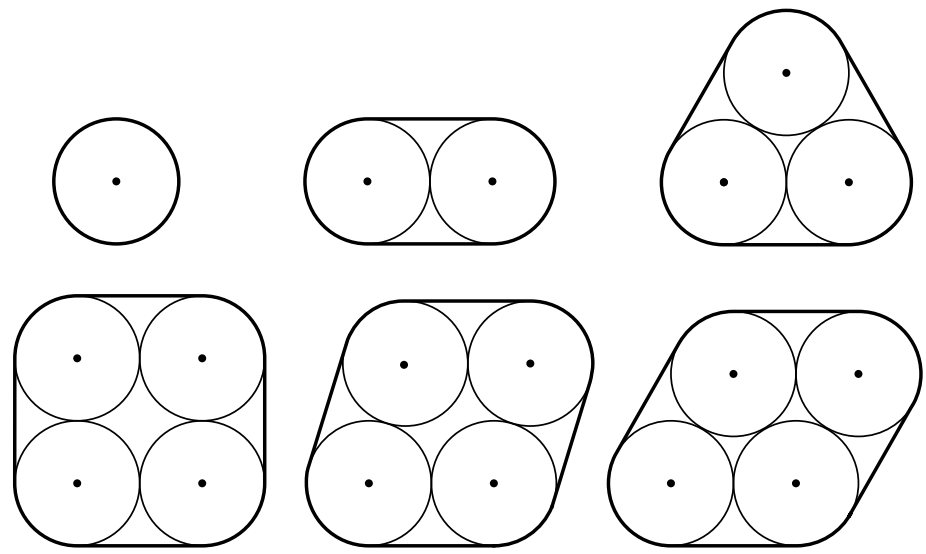

Figure 5: Here we see perimeter-minimizing enclosures of $n=1,2,3$ and 4 unit-diameter disks in the plane. The components in the known minimizing links are outer parallels to such curves at distance $1 / 2$. When $n=4$, the minimizer does not have a unique shape; instead there is a one-parameter family of minimizers. In the last shape on the lower left, there is one additional isolated strut, but it carries no force in the balancing measure.

where different components are at distance 1 . There is a strut from each point along each circular arc to the center of that arc (from $C_{i}$ to $s_{i}$ and from $S$ to $c_{i}$ ). There is also one further strut $\left\{c_{1}, c_{2}\right\}$.

Since we know that this configuration is length-minimizing when constrained by linkthickness, these struts, by Corollary 6.3 and Proposition 6.6, must support a balancing measure $\mu$. Conversely, exhibiting such a measure will re-prove that this configuration is critical for the link-ropelength problem, though to re-prove it is a local minimum would require some second-order theory. We now provide such a measure, which will be a useful comparison of the results of this paper against the results of [4].

Except for $c_{i}$, each point $x$ along the component $C_{i}$ is part of a unique strut $\left\{x, s_{i}\right\}$. The measure assigned to struts in this "wheel" must exactly balance the curvature force $\mathrm{d} \mathcal{K}=N \mathrm{~d} s(x)$ along $C_{i}$. Because the wheel forms a complete circle, at the center points $s_{i}$, the incoming forces from these struts cancel one another, leaving no net force.

The situation on the stadium curve is slightly more complex. The struts from the semicircles of $S$ to the points $c_{i}$ again balance $\mathrm{d} \mathcal{K}=N \mathrm{~d} s(x)$, now for $x$ along the semicircles. Unlike the previous situation, however, these measures have a resultant 

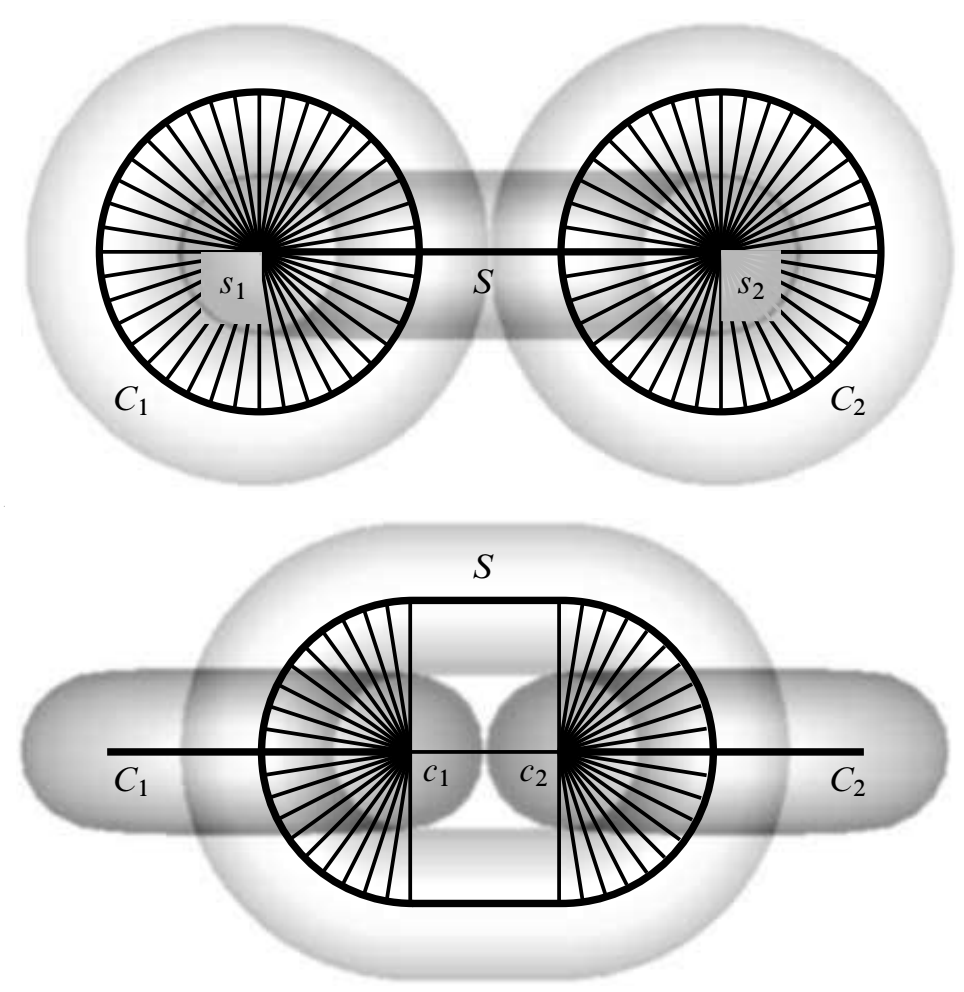

Figure 6: This simple chain is known to be a minimizer for the linkropelength problem, so by the balance criterion, its curvature force must be balanced by some measure on the struts. At the top, we see how the curvature forces along the circular components $C_{i}$ are balanced by the struts coming into the centers $s_{i}$. They produce no net force on either center $s_{i}$. At the bottom, we see how the curvature forces along the semicircles of $S$ are balanced by struts to their centers $c_{i}$. The resulting net inward force on the $c_{i}$ is balanced by an atomic measure on the one remaining strut $\left\{c_{1}, c_{2}\right\}$.

inward force of magnitude 2 at $c_{i}$, directed parallel to the straight segments in the stadium curve. To balance these forces, the measure $\mu$ must have an atom of magnitude 2 at the one remaining strut $\left\{c_{1}, c_{2}\right\}$.

The measure $\mu$ we have described does balance the curvature force everywhere along the link, and thus demonstrates that the link is critical for link-ropelength.

It is worth emphasizing the fact that the inner strut $\left\{c_{1}, c_{2}\right\}$ bears an atom of $\mu$. This stresses the point that in our Kuhn-Tucker theorem and the resulting balance criterion 
we are required to view the Lagrange multiplier $\mu$ as a Radon measure in the dual space $C^{*}(\operatorname{Strut}(L))$, rather than as a density function on struts.

Although ropelength-minimizing, Example 7.1 is not rigid, in the sense that the components $C_{i}$ can be pivoted around the points $c_{i}$ to be centered at any points $s_{i}$ on the semicircles of $S$.

A stronger form of nonuniqueness is exhibited by the minimizing configurations [4] of the five-component link $H\left(T_{4}\right)$, with one component linked to all four others. Here the central component does not even have a uniquely determined shape. Instead there is a one-parameter family of minimizing shapes, corresponding to the deformation seen in Figure 5 for $n=4$. Again, each of the minimizers can be balanced. (As we have proven, the existence of the balancing measure $\mu$ is equivalent to strong criticality for the ropelength problem, but it does not imply that the critical point is isolated.)

For $n>5$, we expect that similar configurations of $H\left(T_{n}\right)$, like the one shown in Figure 7 for $n=6$, are again minimizing. Our balance criterion lets us show they are at least critical:

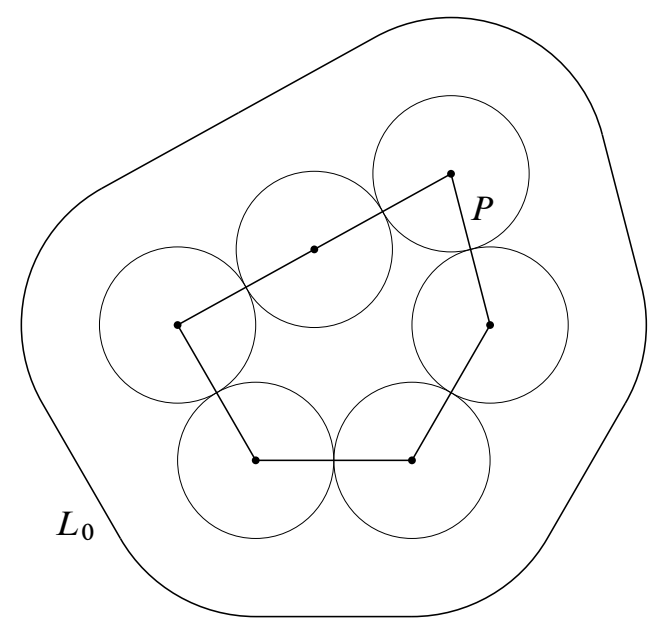

Figure 7: This configuration of $H\left(T_{6}\right)$ is critical for the link-ropelength problem. It is also presumably the minimizer, even though it does not minimize the length of the long component $L_{0}$ alone. That component is the outer parallel at distance 1 to a convex planar polygon $P$. Each other component $L_{i}$ is a unit circle passing through a vertex of $P$ and lying in a perpendicular plane. We have drawn the unit-diameter disks around these vertices, where the thick tubes around the $L_{i}$ intersect the plane inside $L_{0}$. 
Proposition 7.2 Suppose $P$ is a convex planar $n$-gon with unit-length sides and turning angles in $[0,2 \pi / 3]$. Let $L_{0}$ be the outer parallel at distance 1 from $P$, and let $L_{1}, \ldots, L_{n}$ be unit circles, perpendicular to the plane of $P$, passing through the vertices of $P$, and centered at points on $L_{0}$. Then the link $L=L_{0} \cup L_{1} \cup \cdots \cup L_{n}$ is a configuration of $H\left(T_{n}\right)$ with link-thickness 1 that is critical for link-ropelength.

Proof As in the simple chain, each circle $L_{i}$ focuses a wheel of struts to its center point on $L_{0}$, and a measure assigning force $\mathrm{d} s$ to these struts balances the curvature force on each circle while exerting no net force on $L_{0}$.

Let $c_{i}$ be the vertex of $P$ on $L_{i}$, and let $2 \alpha_{i}$ be the turning angle of $P$ there. The condition $\alpha_{i} \leq \pi / 3$ exactly suffices to know that no two vertices (and thus no two $L_{i}$ ) are at distance less than 1 from each other, confirming that LThi $(L)=1$. The curve $L_{0}$ includes an arc of the unit circle around $c_{i}$; from this arc of length $2 \alpha_{i}$ a fan of struts converge to $c_{i}$. To balance the curvature force on $L_{0}$, these struts again have measure equal to $\mathrm{d} s$, giving a net inward force of $2 \sin \alpha_{i}$ on $c_{i}$. The remaining, isolated struts of $L$ connect successive $c_{i}$ along the edges of $P$. Unit atoms of compressive force on these isolated struts produce exactly the outward forces $2 \sin \alpha_{i}$ at $c_{i}$ needed to balance the inward forces from $L_{0}$.

By Corollary 6.3, the existence of this balancing measure on the struts proves that $L$ is critical.

For $n \leq 5$, we know these configurations for $H\left(T_{n}\right)$ are ropelength minimizers. For $n>5$, the component $L_{0}$, having length $n+2 \pi$, is longer than it needs to be: at the expense of lengthening some other components, it could be shortened to length $P_{n}$, which, asymptotically, is much smaller, being $O(\sqrt{n})$. However, calculations we have done suggest that the tradeoff is not worthwhile and so the critical configuration described above is probably the global minimum for ropelength.

The examples given in Proposition 7.2-critical configurations and presumed minimizers for $H\left(T_{n}\right)$-are quite interesting. The shape of $L_{0}$ is free to move in an $(n-3)$-parameter family; each other component is free to pivot (about its vertex of $P$ and along one of the arcs of $L_{0}$ ), giving an additional $n$ parameters for the shape of the whole link $L$. We also note that these examples are tight links that are not packed tightly: Consider the thick (unit diameter) tube around one of these configurations. As $n$ increases, it occupies an ever smaller fraction of the volume of its convex hull. This should be compared with experiments of Millett and Rawdon [23] on this volume fraction.

Although we have stated Proposition 7.2 above only for stars $T_{n}$, the same balancing works for the links $H(T)$ based on other trees $T$. Each component linked to $n$ others 
should have the shape of $L_{0}$ above. We note, however, that critical links built in this way are not always minimizers.

Example 7.3 Consider the tree $T_{n, m}$ with $n+m$ vertices, including one of valence $n$ connected to another of valence $m$. The link $H\left(T_{n, m}\right)$ then has two long components, $L_{0}$ and $L_{1}$, linked to each other and to $n-1$ and $m-1$ short components, respectively. For large enough $n$ and $m$, we can construct two thick versions of $H\left(T_{n, m}\right)$, called the $A$ and $B$ configurations, as follows.

For the $A$ configuration, we follow Proposition 7.2 to build $L_{0}$ and $L_{1}$ as outer parallels to convex planar $n$ - and $m$-gons $P_{0}$ and $P_{1}$ in perpendicular planes. If $n=2 k$, then we let $P_{0}$ take the shape of a $(k-1) \times 1$ rectangle capped with equilateral triangles on both short sides; if $n=2 k+1$, we omit one triangle. (The precise shape is unimportant, but we need at least one sharp angle on each polygon.) Further, we choose the tip of such a triangle as the vertex of $P_{0}$ corresponding to $L_{1}$ (and as the vertex of $P_{1}$ corresponding to $L_{0}$ ). Then it is straightforward to check that the other components stay sufficiently far from each other for this $A$ configuration to indeed have LThi $=1$; its total length $(2 \pi+1)(n+m)$.

However, when $n$ and $m$ are large enough, we can save two units of length as follows. Construct a tight configuration of $H\left(T_{n-1}\right)$ as in Proposition 7.2, using a regular polygon. (Again, the precise shape is not important, but here we need a large hole in the middle of the polygon.) This configuration, and indeed the unit-diameter thick tubes around its components, is contained in a round solid torus $U_{n}$ of minor radius $3 / 2$ and major radius $1+1 /(2 \sin \pi / n-1)$. Then construct the analogous configuration of $H\left(T_{m-1}\right)$ contained in a solid torus $U_{m}$. Finally, place these two pieces in space so that $U_{n}$ and $U_{m}$ form a (loose) Hopf link. (This is possible as long as the major radii are at least 3 , corresponding to $n, m \geq 14$.) The resulting link is the $B$ configuration of $H\left(T_{n, m}\right)$. Because the large Hopf link is loose, there are no struts from $H\left(T_{n-1}\right)$ to $H\left(T_{m-1}\right)$. Since each of these pieces is balanced, so is the $B$ configuration of $H\left(T_{n, m}\right)$.

If, as we believe, these $B$ configurations are the ropelength minimizers, then they are the first ones known in which certain pairs of linked components are not in contact. (We note that the same must be true for the $n$-component Hopf links for large $n$, since their minimum ropelength [3] is $O\left(n^{3 / 2}\right)$. There, however, no explicit candidate minimizer is known. And our $B$ configuration here has the additional property that certain linked pairs are not even connected by chains of touching components.)

In all of the examples $H(T)$ discussed above, each component is a convex plane curve built from straight segments and arcs of unit circles. The proven minimizers are 
minimizers in their isotopy class for the original ropelength problem [4], as well as minimizers in their link-homotopy class for link-ropelength. In fact, in [2] we will consider a family of thicknesses with varying stiffness. Each of these thicknesses is characterized by a stiffness $\lambda$, meaning a lower bound on the diameter of curvature for a unit-thickness curve. Our $H(T)$ are ropelength-minimizers for the whole family, as long as the stiffness $\lambda$ does not exceed 2 , when circular arcs of larger diameter would be needed. We will also develop an analog of our balance criterion for these other ropelength problems, and will see that all the $H(T)$ discussed above (including those that are not minimizers) are critical for all formulations of ropelength where $\lambda \leq 2$.

\subsection{Local minima for ropelength}

We do not attempt in the paper to discuss second-order behavior of ropelength near a critical point - in particular we have no way yet to distinguish between local minima and saddle points for this problem. Of course, the known minimizers must be local minima, and it is also easy to give critical configurations which are not local minima, as in Example 7.4 below.

Many researchers have used numerical simulations of the ordinary ropelength problem to look for nontrivial local minima for knots, in particular for the unknot. Such configurations have been termed Gordian unknots since they can be untangled topologically but not physically. Pieransky et al [29] have numerically simulated a reasonable candidate for a Gordian unknot, but we are very far from being able to prove its existence.

In Example 7.3 we gave two distinct critical configurations for $H\left(T_{n, m}\right)$, and we expect that this will lead to the provable existence of two distinct local minima. In particular, our investigations lead us to predict that one cannot move from the $A$ configuration of length $(2 \pi+1)(n+m)$ to the suspected global minimum $B$ without first increasing ropelength. This shows there must be a second local minimum; we expect, however, that this is not $A$ but instead a third configuration of intermediate length.

This connects back to Alexander Nabutovsky's original work on ropelength in higher dimensions and codimensions [27]. He showed using recursive function theory that, in those higher dimensions, a ropelength constraint often introduces new components into the moduli space of unknotted hyperspheres; in particular there are infinitely many local minima for ropelength. While for two-spheres in $\mathbb{R}^{3}$ or for circles in $\mathbb{R}^{2}$ there are presumably no such minima, we do expect there must be infinitely many Gordian unknots in $\mathbb{R}^{3}$. Our two critical configurations of $H\left(T_{n, m}\right)$ are perhaps a first step toward proving this. 


\subsection{Elastic tension energies}

All of the links presented above are critical or minimizing for the sum of the lengths of their components. This is a beautiful functional, but it is physically somewhat unrealistic: elastic ropes should minimize a quadratic functional of the form

$$
\sum_{i} a_{i}\left(\operatorname{Len}\left(L_{i}\right)-\ell_{i}\right)^{2}
$$

where $a_{i}>0$ is the elasticity and $\ell_{i}$ the rest length of the $i$-th component. Criticality for this functional is equivalent to that for $\sum t_{i} \operatorname{Len}\left(L_{i}\right)$ where the tension $t_{i}$ is $t_{i}=2 a_{i}\left(\operatorname{Len}\left(L_{i}\right)-\ell_{i}\right)$. Assuming these tensions are nonnegative (that is, that no component's length is less than its rest length) our balance criterion extends immediately to handle this case: the strut force $A_{\mathrm{S}}^{*}(\mu)$ must balance the tension-weighted curvature force $\sum t_{i} \mathcal{K}_{i}$.

In the known minimizing links, such as the simple chain, each component separately achieves its minimal possible length. Thus these examples also minimize all elastic energies with nonnegative tensions $t_{i} \geq 0$.

This behavior, however, seems rather exceptional. The examples in Proposition 7.2 do not minimize all such functionals. In particular, if the tension in the long component is large enough, it will shrink to length $O(\sqrt{k})$ while some of the shorter components gain length.

Also in the Borromean rings, if the three components have different tensions, the configuration we describe below (Section 10) would no longer be critical. Similarly, clasps (Section 9) in which the two ropes have different tensions again have new critical configurations. In [37] we describe in detail the shapes of these asymmetric clasp curves, as well as their appearance in more complicated clasp-like links even when tensions are equal. (Note that link-thickness was called Gehring thickness there, as in early drafts of this paper.)

\subsection{Nonembedded critical links}

To illustrate the differences between the Gehring problem and the original ropelength problem, we now give some examples of a different flavor: critical configurations that are nonembedded and thus have infinite ropelength in the original sense.

Any knot is of course link-homotopic to the unknot. The link-ropelength minimizer degenerates to a point (of length zero). The same happens for any component of an arbitrary link that is link-homotopically split from the rest of the link. 
Milnor showed that, up to link homotopy, links of two components are classified by their linking number [25].

Example 7.4 When the linking number is zero, the components split, and the linkropelength minimizer degenerates to have length zero. We can, however, also describe another critical configuration for this unlink: one component degenerates to a point $p$ while the second is a unit circle centered at $p$. This is clearly an unstable critical point: obvious deformations can decrease the ropelength to second order.

The case of linking number 1 is close to Gehring's original problem: the minimizer is the same Hopf link built from round circles. (This case fits in the class $H\left(T_{n}\right)$ considered above.) For larger linking number, we can use Corollary 6.3 to exhibit many critical configurations as follows:

Example 7.5 For linking number $m n$ there is a critical configuration $L_{m, n}$ consisting of the minimizing Hopf link with one component covered $m$ times and the other covered $n$ times. Its total length is thus $2 \pi(m+n)$. There are other critical configurations, sometimes shorter. For example, each component can be a figure-eight built from two tangent circles. Figure 8 shows a configuration like this with total length $2 \pi(m+n)$ and linking number $m n-m_{1} n_{1}$. The best configurations we know for linking number 17 , for instance, use $(m, n)=(6,3)$ or $(4,5)$. Assuming configurations like these are the minimizers for two-component links, they give examples where the set of minimizers is disconnected (since we can interchange the two components, or reorder the way one component covers its figure-eight).

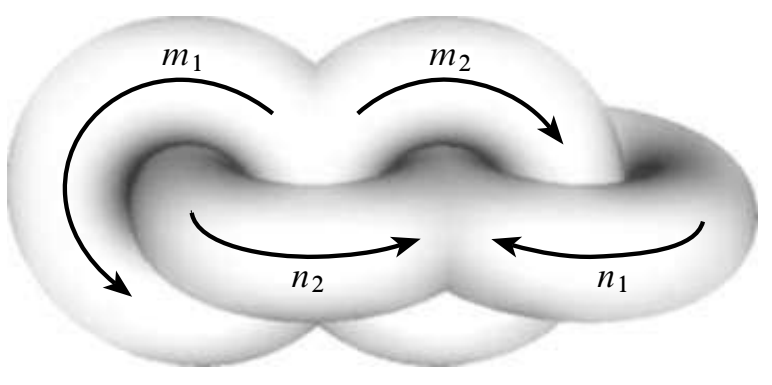

Figure 8: In this configuration of two curves from Example 7.5, each circle is covered $m_{i}$ or $n_{i}$ times, as labeled. If $m=m_{1}+m_{2}$ and $n=n_{1}+n_{2}$, then the curve has total length $2 \pi(m+n)$, and linking number $m n-m_{1} n_{1}$. It is constrained-critical, though often not minimal, for the Gehring problem in the link-homotopy class defined by its linking number. 
None of these configurations is embedded, so they are not critical points for the original ropelength problem: as expected, the extra freedom in the Gehring problem sometimes allows for shorter solutions. As a further example, consider the $(2,4)$-torus link, with linking number 2 . We have computed the presumed ropelength-minimizer numerically, as in [35]. The results are shown in Figure 9; this solution is longer than the covered Hopf link $L_{2,1}$ (the presumed link-ropelength-minimizer) and is not even critical for the Gehring problem.

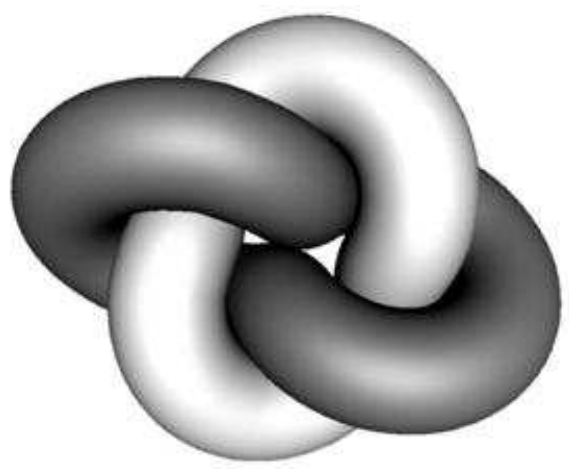

Figure 9: This picture shows a numerically computed minimizer for the original ropelength problem on the $(2,4)$-torus link. Because it has a strut between two points on the same component (shown center, where the darker tube contacts itself) that carries nonzero force, it is not balanced for the Gehring problem considered here. It is longer than $L_{2,1}$, the Hopf link with one component doubly covered, which we conjecture is the minimizer for link-ropelength in this link-homotopy type. Notice that both of these configurations break the symmetry between the components of the link, so we expect to also find a (longer) critical configuration where the two components are congruent.

For links of more than two components, linking numbers do not suffice to distinguish link-homotopy types; we must also consider Milnor's $\mu$-invariants [25; 16]. For instance, the Borromean rings, with no nonzero linking numbers, belong to a nontrivial link-homotopy class because they have $\mu$-invariant equal to 1 .

Numerical experiments performed with Brakke's Evolver (compare [35]) suggest that the minimizing Borromean rings for the link-ropelength problem should consist of three congruent curves in perpendicular planes. In [4], we described such a configuration built from circular arcs of radius 1. Unfortunately, Corollary 6.3 shows this configuration is not even critical for length when constrained by link-thickness. In Section 10, the 
culmination of our paper, we will explicitly describe a very similar configuration of the Borromean rings, which we prove is critical and believe is the minimizer.

However, in order to solve for these Borromean rings, we must first consider a simpler interaction between two ropes: the clasp that occurs when one rope is pulled over another. Describing this will require a notion of generalized links.

\section{Generalized link classes}

Although some of our definitions have applied to arbitrary curves, so far we have been treating only ordinary (closed) links. We now want to consider generalized problems involving curves with endpoints. To get meaningful link classes in this setting, we must include constraints for the endpoints and obstacles for the link.

Definition A generalized link $L$ is a curve $L$ (with disjoint components $L_{1}, \ldots, L_{N}$ ) together with obstacles and endpoint constraints. In particular, each endpoint $x \in \partial L$ is constrained to stay on some affine subspace $M_{x} \subset \mathbb{R}^{3}$, which can have dimension 0 , 1 or 2. Furthermore, there is a finite collection of obstacles for each component $L_{i}$ of the link. Each obstacle

$$
\left\{p \in \mathbb{R}^{3}: g_{i j}(p)<0\right\}
$$

is given by a $C^{1}$ function $g_{i j}$ with 0 as a regular value. By calling them obstacles, we mean that $L_{i}$ is constrained to stay in the region where $\min _{j} g_{i j} \geq 0$.

While we could allow even more general endpoint and obstacle constraints, this version fits nicely with our overall setup, and allows for all the specific examples we have in mind.

Definition Suppose $L=\bigcup_{i} L_{i}$ is a generalized link, with obstacles $g_{i j}$ and endpoint constraints $M_{x}$. Then its link-homotopy class $[[L]]$ is the set of all links $L^{\prime}$ that are link-homotopic to $L$ through links where each component avoids its obstacles and maintains its endpoint constraints. (As before, in a link homotopy, each component of $L$ can intersect itself but not the others.)

This definition is comparable to our previous definition for closed links (Section 2); as in the discussion at the end of Section 6, we may restrict our attention to tame link classes.

Given a generalized link $L$, only variations preserving the endpoint constraints should be allowed. A vector field $\xi \in \operatorname{VF}(L)$ is said to be compatible with the constraints if 
it is tangent to $M_{x}$ at each endpoint $x \in \partial L$. We write $\operatorname{VF}_{c}(L)$ for the space of all compatible vector fields.

Given a set of obstacles $g_{i j}<0$ and a link $L=\bigcup L_{i}$, we write

$$
O(L):=\min _{i, j} \min _{x \in L_{i}} g_{i j}(x) .
$$

Then $L$ avoids the obstacles $g_{i j}$ if and only if $O(L) \geq 0$. We define the wall struts of $L$ by

$$
\operatorname{Wall}_{i j}(L):=L_{i} \cap\left\{g_{i j}=0\right\}, \quad \operatorname{Wall}(L):=\bigsqcup_{i, j} \operatorname{Wall}_{i j}(L) .
$$

This incorporates those parts of $L$ on the boundary of the obstacle, but is not strictly speaking a subset of $L$ since one point $x \in L_{i}$ might be in several of the Wall $i j$. When $O(L)=0$, by Clarke's Theorem 3.1 we have

$$
\delta_{\xi}^{+} O(L)=\min _{i, j} \min _{x \in \operatorname{Wall}_{i j}(L)}\left\langle<\xi_{x}, \nabla g_{i j}\right\rangle
$$

Again, we collect the various derivatives appearing on the right-hand side into a rigidity operator $A_{\mathrm{W}}: \mathrm{VF}_{c}(L) \rightarrow C(\mathrm{Wall}(L))$ on wall struts, given by

$$
A_{\mathrm{W}} \xi(x):=\left\langle\xi_{x}, \nabla g_{i j}\right\rangle
$$

when $x \in$ Wall $_{i j}$. Its adjoint $A_{\mathrm{W}}^{*}$ is then

$$
\int_{L} \xi \mathrm{d} A_{\mathrm{W}}^{*}(\mu)=\int_{\text {Wall }(L)} A_{\mathrm{W}} \xi \mathrm{d} \mu=\sum_{i, j} \int_{x \in \operatorname{Wall}_{i j}(L)}\left\langle\xi_{x}, \nabla g_{i j}\right\rangle \mathrm{d} \mu(x) .
$$

We also have corresponding definitions for locally minimal, strongly critical, and critical configurations of $L$ :

Definition We say that a generalized link $L$ is a local minimum for length when constrained by LThi if we have $\operatorname{Len}\left(L^{\prime}\right) \geq \operatorname{Len}(L)$, for all sufficiently $C^{0}$-close links $L^{\prime}$ with the same obstacle and endpoint constraints and with $\operatorname{LThi}\left(L^{\prime}\right) \geq \operatorname{LThi}(L)$. We say $L$ is strongly critical (respectively, is critical) for minimizing length when constrained by LThi if there is $\varepsilon>0$ such that for all compatible smooth $\xi$ with $\delta_{\xi}$ Len $=-1$, the quantity

$$
\min \left(\delta_{\xi}^{+} \operatorname{LThi}(L), \delta_{\xi}^{+} O(L)\right)
$$

is at most $-\varepsilon$ (respectively, is negative). 
As in our discussion of Kuhn-Tucker at the beginning of Section 5, these notions will be equivalent only under a regularity assumption corresponding to the constraint qualification of Mangasarian and Fromovitz [20]:

Definition A generalized link $L$ is LThi-regular if there is a thickening field, meaning a smooth compatible $\eta$ for which $\delta_{\eta}^{+} \operatorname{LThi}(L)>0$ and $\delta_{\eta}^{+} O(L)>0$.

Note that, while we require $\eta$ to strictly increase LThi and to move away from the obstacles, both to first order, there is no corresponding requirement for the endpoint constraints, since they are linear equality constraints instead of nonlinear inequality constraints.

We can now prove a generalization of Proposition 6.6:

Proposition 8.1 If a generalized link $L$ is a LThi-regular local minimum when constrained by LThi, then $L$ is critical. Also, if $L$ is LThi-regular and critical when constrained by LThi, then it is strongly critical.

Proof The regularity of $L$ means there exists a thickening field $\eta \in \operatorname{VF}_{c}(L)$. We may assume $\delta_{\eta} \operatorname{Len}(L) \geq 0$ for otherwise $L$ is neither minimal nor critical; we then scale $\eta$ so that $\delta_{\eta} \operatorname{Len}(L)<1$.

Suppose that $L$ is a local minimum but not critical. Then for some compatible vector field $\xi$ we have $\delta_{\xi} \operatorname{Len}(L)<0$ while $\delta_{\xi}^{+} \operatorname{LThi}(L) \geq 0$ and $\delta_{\xi}^{+} O(L) \geq 0$. For small $t>0$, consider the links $L_{t}=L+t(\xi+\epsilon \eta)$. Then

$$
\left.\frac{\mathrm{d} \operatorname{Len}\left(L_{t}\right)}{\mathrm{d} t^{+}}\right|_{t=0}=\delta_{\xi} \operatorname{Len}(L)+\epsilon \delta_{\eta} \operatorname{Len}(L) .
$$

We choose $0<\epsilon<-\delta_{\xi} \operatorname{Len}(L) / \delta_{\eta} \operatorname{Len}(L)$, so this derivative is negative at time 0 . Thus for small $t$, the $L_{t}$ have length less than Len $(L)$, contradicting minimality if they obey our constraints. But

$$
\left.\frac{\mathrm{d} \operatorname{LThi}\left(L_{t}\right)}{\mathrm{d} t^{+}}\right|_{t=0}>0,\left.\quad \frac{\mathrm{d} O\left(L_{t}\right)}{\mathrm{d} t^{+}}\right|_{t=0}>0,
$$

and the endpoint constraints are linear, so the links $L_{t}$ meet all our constraints for small $t>0$.

Now suppose that $L$ is critical without being strongly critical. Then there exists a sequence of compatible vector fields $\xi_{i} \in \mathrm{VF}_{c}(L)$ with $\delta_{\xi_{i}} \operatorname{Len}(L)=-1$ but with either $\delta_{\xi_{i}}^{+}$LThi $(L) \rightarrow 0$ or $\delta_{\xi_{i}}^{+} O(L) \rightarrow 0$. Then we observe that $\delta_{\eta+\xi_{i}} \operatorname{Len}(L)<0$ for all $i$, while by Corollary 3.3 either

$$
\lim \delta_{\eta+\xi_{i}}^{+} \text {LThi } \geq \delta_{\eta}^{+} \text {LThi }>0
$$


or

$$
\lim \delta_{\eta+\xi_{i}}^{+} O \geq \delta_{\eta}^{+} O>0 .
$$

Taking $i$ large enough that one of these quantities is positive, we get a contradiction to the criticality of $L$.

So far, this development has paralleled that of Section 6; we now diverge from our previous course. Earlier, we saw that every closed link is LThi-regular: rescaling always provides a thickening field. In the generalized setting, this is no longer the case. Thus minimality no longer implies criticality.

Example 8.2 To give a specific example, rotate the constraints of Example 5.1 around the $z$-axis to give obstacles $g_{1}=\left(x^{2}+y^{2}-1\right)^{3}-z<0$ and $g_{2}=z<0$ for an unknot $L$. The unit circle in the $x y$-plane is on the boundary of both obstacles, and is clearly the minimum-length configuration in its homotopy class. However, it is not critical: shrinking it toward the origin will reduce its length to first order; the constraint $g_{1} \geq 0$ is now violated, but not to first order.

Further, criticality and strong criticality may be different: if we allowed infinitely many obstacles, we could construct critical, but not strongly critical links by following the lead of Example 5.6. (If we do not allow infinitely many obstacles, then an open question remains: is strong criticality a stronger assertion than criticality?)

Example 8.3 To justify our emphasis on strong criticality (rather than restricting our attention to regular, critical links) we also note that it is easy to construct strongly critical links that are not regular; simply take $L$ to be the unit circle in the $x y$-plane, with constraints $g_{1}(x, y, z)=x^{2}+y^{2}-1$ (so the excluded region is the infinite cylinder around the $z$-axis) and $g_{2}=-g_{1}$. This link is trapped on the cylinder $g_{1}=0=g_{2}$, so it has no thickening field. On the other hand, it is clearly strongly critical.

Now we are ready to extend our balance theorem to the generalized setting. We will accommodate the endpoint constraints by restricting our attention to compatible vector fields. Our other constraints are then Dist $\geq 1$ on $L^{(2)}$ and $g_{i j} \geq 0$ along $L_{i}$. The set $Y$ of active constraints then consists of the struts together with the wall struts.

Theorem 8.4 A generalized link $L$ is strongly critical for link-ropelength if and only if there is a positive Radon measure $\mu$ on $\operatorname{Strut}(L) \sqcup \mathrm{Wall}(L)$, such that

$$
-\mathcal{K}=\left(A_{\mathrm{S}} \oplus A_{\mathrm{W}}\right)^{*} \mu
$$

as linear functionals on $\operatorname{VF}_{c}(L)$. This means that $-\mathcal{K}$ and $\left(A_{\mathrm{S}} \oplus A_{\mathrm{W}}\right)^{*} \mu$ agree as forces along $L$ except at endpoints $x \in \partial L$, where they may differ by an atomic force in a direction normal to $M_{x}$. 
Proof This is again a straightforward application of our Theorem 5.4, using

$$
X=\operatorname{VF}_{c}(L), \quad Y=\operatorname{Strut}(L) \sqcup \operatorname{Wall}(L), \quad f=\delta \text { Len }, \quad A=A_{\mathrm{S}} \oplus A_{\mathrm{W}} .
$$

Remark 8.5 Remember that $\mathcal{K}$ has been defined to include an inward-pointing atom at each endpoint $x \in \partial L$. We can ignore these, however, when applying this theorem, as long as the link $L$ meets each endpoint constraint $M_{x}$ normally. We know of no examples of critical links where this is not the case.

The regularity described in Corollary 6.2 and Proposition 6.5 carries over to generalized links:

Proposition 8.6 If the generalized link $L$ is strongly critical for link-ropelength, then $L$ is FTC and $C^{1}$.

Proof The proof follows that of Corollary 6.2 and Proposition 6.5. From equation (8-1), we find that $A_{\mathrm{W}}^{*} \mu$ has distributional order zero just like $A_{\mathrm{S}}^{*} \mu$, so $L \in \mathrm{FTC}$ follows immediately from the balance criterion of Theorem 8.4.

Now suppose $L$ is not $C^{1}$ but instead has some corner $x$ with $\mathcal{K}\{x\} \neq 0$. By Theorem 8.4, this curvature force is balanced by struts and wall struts. So there is at least one strut or wall strut acting on $x$ in a direction with negative inner product with $\mathcal{K}\{x\}$. In the case of a strut $\{x, y\}$, we refer again to Figure 3: some points near $x$ along $L$ would be nearer to the endpoint $y$. But similarly, in the case of a wall strut, we have $\left\langle\mathcal{K}, \nabla g_{i j}\right\rangle<0$, but this means that some points near $x$ along $L$ violate this obstacle constraint. In either case, we get the desired contradiction.

To understand the interplay between struts and wall struts, we now offer a simple example of a generalized link $L$ with nonempty boundary which is balanced, needing nonzero force on the wall struts.

Example 8.7 Cut the simple chain of Figure 6 by parallel planes through $s_{1}$ and $s_{2}$ with normal vector $c_{1}-c_{2}$, and let $L$ be the part of the chain lying between the two planes. This generalized link includes two semicircles with endpoints normal to the planes, and also the inner stadium curve, which is tangent to the planes at $s_{1}$ and $s_{2}$. We let the planes bound an obstacle, forcing $L$ to stay between the planes, and we use them also as endpoint constraints. Then $L$ is balanced: though the semicircles now exert a net outward force on $s_{1}$ and $s_{2}$, this is balanced by wall struts at these points. And the internal balance for the stadium curve remains the same. 


\section{The tight clasp}

The tight configurations of Section 7 were the simplest closed links we could imagine: the Hopf link, and various connect sums of Hopf links in which each component is still a convex plane curve. But there is an even simpler interaction between two ropes, the clasp formed when one rope is pulled taut over another, as at the junctions of a woven net, or when a bucket is lifted from a well by passing a rope through its rope handle. We can model a single clasp as a generalized link with endpoint constraints.

To define the simple clasp, fix two parallel planes $P$ and $\widetilde{P}$ at least 2 units apart. Then take two unknotted $\operatorname{arcs} \gamma$ and $\tilde{\gamma}$ that lie between the planes, with the endpoints of $\gamma$ constrained to lie in $P$ and those of $\tilde{\gamma}$ in $\widetilde{P}$. Let the halfspace bounded by $P$ that does not include $\widetilde{P}$ be an obstacle for the component $\tilde{\gamma}$, and vice versa, and select the isotopy class of such links shown in Figure 10. This is the class where closing each arc in the plane of its endpoints would produce a Hopf link.

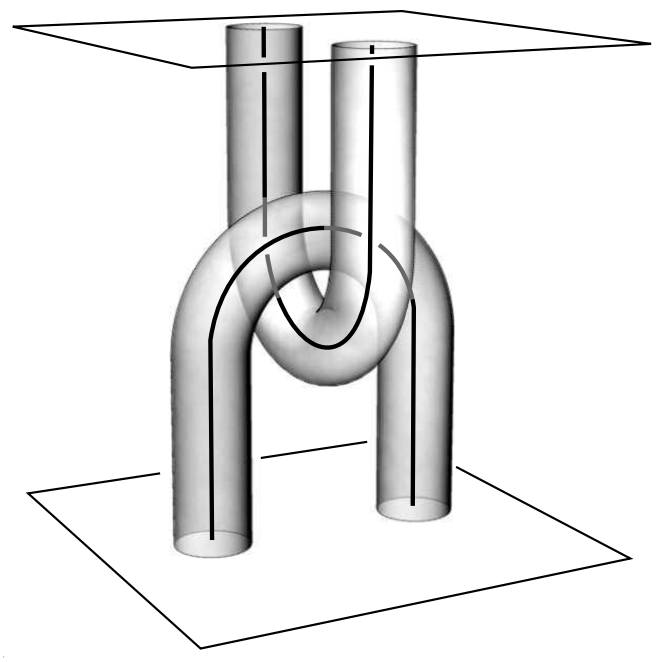

Figure 10: The simple clasp has two components, one attached at both ends to the ceiling and the other to the floor, linked with one another as shown. The configuration shown, with each component consisting of a semicircle joined to two straight segments, is neither critical nor minimal.

It is natural to assume that the minimizing configuration for this problem would consist of semicircular arcs passing through each others' centers, together with straight segments joining the semicircles to the constraint planes, much like the Hopf chain of Example 7.1. But this naive clasp is not balanced: each semicircle focuses its curvature force on the tip of the other, and there is no way to balance these forces (as the isolated 
strut carrying an atom of compressive force did in the Hopf chain). The naive clasp is thus not minimizing, though we will see it is very close: the critical configuration we construct here is only half a percent shorter.

Example 9.1 Suppose the horizontal planes $P$ and $\widetilde{P}$ in the definition of the simple clasp are taken instead to be only one unit apart. Consider the configuration where the curves $\gamma$ and $\tilde{\gamma}$ are semicircles in perpendicular vertical planes. The curvature of each semicircle can be balanced by uniform strut tension, transmitting a net vertical force to the tip of the other semicircle. That vertical force can be balanced by a wall strut at each tip. Therefore, this configuration is critical for link-ropelength.

In the case of interest, where $P$ and $\widetilde{P}$ are far apart and there are no wall struts to balance the tips, we must look harder for a solution. We will now construct critical configurations, constrained by the link-thickness LThi, for the simple clasp problem and for a family of related problems where the ends of the ropes are pulled outward as in Figure 11. These solutions minimize length under natural symmetry assumptions, and we believe they are the global minimizers even without imposed symmetry. Below in Section 10, we will construct a critical configuration of the Borromean rings that contains portions of these clasp curves. Thus, a thorough understanding of these generalized links will aid us in understanding that more complicated closed link.

\subsection{Symmetry conditions and a convenient parametrization}

We describe configurations of the clasp where the two components are congruent plane curves, lying in planes perpendicular to each other and to the constraint planes. To fix these symmetries in coordinates, let the constraints be the planes $z= \pm C$, and let the component $\gamma$ lie in the $x z$-plane while $\tilde{\gamma}$ lies in the $y z$-plane. The clasp has mirror symmetry across each of these planes (preserving each component). It also has a symmetry interchanging the two components, which we denote $p \mapsto \tilde{p}$, given by fourfold rotation about the $z$-axis together with reflection across the $x y$-plane. These symmetries generate a point group of order eight in $O(3)$ whose Conway-Thurston orbifold notation $[9 ; 10]$ is $2 * 2$. Algebraically it is isomorphic to $D_{4}$.

The argument we present below to derive the critical clasps for the Gehring problem can easily be extended to show these are the unique critical configurations among curves with this $2 * 2$ symmetry. We omit the details, however, because we know of no way to show that the overall minimizers must have this symmetry. If one could prove this, it would then follow that our clasps are the minimizers.

Our symmetry assumptions mean that the clasp is described by the shape of half of the component $\gamma$, from its tip along the $z$-axis into the $x>0$ half-plane and up to the 
plane $P$. This consists of a curved arc near the tip joined to a straight segment near $P$. Since the curved arc is strictly convex, we can parametrize it by the angle $\varphi$ made by its tangent vector above the horizontal, as in Figure 12. In fact, we will use the sine of this angle, $u=\sin \varphi$, as our parameter. Thus in the simple clasp, for $u \in[0,1]$ we write

$$
\begin{aligned}
& \gamma( \pm u)=( \pm x(u), 0, z(u)), \\
& \tilde{\gamma}( \pm u)=(0, \pm x(u),-z(u)) .
\end{aligned}
$$

Elementary calculations show the following:

Lemma 9.2 For a convex curve $\gamma$ in the $x z$-plane, parameterized by the sine $u$ of its direction $\varphi \in\left[-\frac{\pi}{2}, \frac{\pi}{2}\right]$, the arclength $s$ satisfies

$$
\mathrm{d} s=\sec \varphi \mathrm{d} x=\csc \varphi \mathrm{d} z=\frac{\mathrm{d} u}{\kappa \sqrt{1-u^{2}}},
$$

where the curvature $\kappa$ is given by

$$
\kappa=\frac{\mathrm{d} \varphi}{\mathrm{d} s}=\frac{\mathrm{d} u}{\mathrm{~d} x}
$$

For the simple clasp described above, each component turns a total of $180^{\circ}$, meaning that $u$ ranges from -1 , through 0 at the tip, to 1 . We can also consider more general clasp problems where the four ends of rope are not vertical (being attached to horizontal planes) but instead are pulled out at some angle (being attached to tilted planes).

Given $0 \leq \tau \leq 1$, we define the $\tau$-clasp to be a problem like the simple clasp where the arc $\gamma$ starts at $u=-\tau$ and then turns through angle $2 \arcsin \tau$ to reach $u=\tau$. Our critical $\tau$-clasps have the same $2 * 2$ symmetry as the simple clasp. To put the $\tau$-clasp into our framework of generalized links, we constrain the four endpoints to four planes, each making angle $\arcsin \tau$ with the vertical, as in Figure 11. The complement of the wedge formed by the planes containing the endpoints of each arc acts as an obstacle for the other arc. The simple clasp is the $\tau$-clasp with $\tau=1$, where the wedges degenerate to halfspaces.

\subsection{Struts between perpendicular planes}

Whenever two curves in perpendicular planes are connected by a strut, elementary trigonometry gives us first order information about the curves at both endpoints. We state a general lemma, which we will use here for the clasp and again for the Borromean rings. 


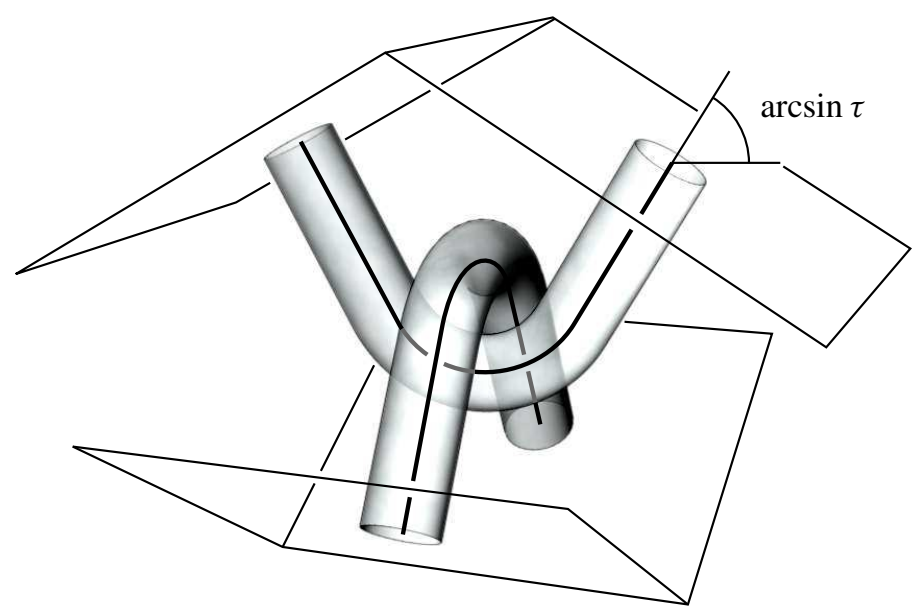

Figure 11: In this variant of the simple clasp problem, the endpoints of the two ropes are constrained to lie in four planes whose normals make angle $\arcsin \tau$ with the horizontal. The parameter $u=\sin \varphi$ ranges from $-\tau$ to $\tau$ along each arc, as shown at the end of the top right arc. If extended, the four planes shown would form the sides of a tetrahedron. Each arc is constrained to lie in the wedge formed by the planes containing the endpoints of the other arc.

Let $P_{1}$ and $P_{2}$ be two planes meeting perpendicularly along a line $\ell$, and let $\gamma_{i} \subset P_{i}$ be two components of a link. At a point $p_{i} \in \gamma_{i}$, we write $x_{i}$ for the distance from $p_{i}$ to $\ell$, and $u_{i}$ for the cosine of the angle between $\ell$ and the line tangent to $\gamma_{i}$ at $p_{i}$. These quantities generalize the $x$ and $u$ of Lemma 9.2 above.

Lemma 9.3 Let $\gamma_{1}$ and $\gamma_{2}$ be two components of a link $L$, lying in perpendicular planes. Suppose there is a strut $\left\{p_{1}, p_{2}\right\}$ of length 1 connecting these components. Then in the notation of the previous paragraph we have $0 \leq x_{i} \leq u_{i} \leq 1$, and any two of the numbers $x_{1}, x_{2}, u_{1}, u_{2}$ determine the other two, according to the formulas

$$
\begin{aligned}
& x_{i}^{2}=1-\frac{x_{j}^{2}}{u_{j}^{2}}=\frac{u_{i}^{2}\left(1-u_{j}^{2}\right)}{1-u_{i}^{2} u_{j}^{2}}, \\
& u_{i}^{2}=\frac{1-x_{j}^{2} / u_{j}^{2}}{1-x_{j}^{2}}=\frac{x_{i}^{2}}{1-x_{j}^{2}},
\end{aligned}
$$

where $j \neq i$.

Proof Picking cartesian coordinates such that $\ell$ is the $z$-axis and $P_{i}$ are coordinate planes, we find the strut difference vector $p_{1}-p_{2}$ is $\left(x_{1}, x_{2}, \Delta z\right)$, for some number $\Delta z$. 
Since this strut has length 1 and is perpendicular to each $\gamma_{i}$, we have

$$
\Delta z^{2}+x_{1}^{2}+x_{2}^{2}=1, \quad \Delta z=x_{i} \frac{u_{i}}{\sqrt{1-u_{i}^{2}}} .
$$

Simple algebraic manipulations, eliminating $\Delta z$, lead to the equations given.

Note that the condition $x_{i} \leq u_{i}$ is exactly the condition that the unit normal circle around $p_{i}$ intersects $P_{j}$; the two points of intersection are mirror images (across $P_{i}$ ), with the same $x_{j}$ and $u_{j}$ values. Also note that we don't need to have $\gamma_{i} \subset P_{i}$ in the lemma; it suffices that $\gamma_{i}$ be tangent to $P_{i}$ at $p_{i}$.

Whenever we have a pair of curves in perpendicular planes, which stay a constant distance 1 apart, we can apply this lemma everywhere along the curves. Each curve $\gamma_{i}$ is determined as the intersection of the plane $P_{i}$ with the unit-radius tube around the other curve $\gamma_{j}$. This will be the situation for the clasp.

\subsection{The balancing equations for the clasp}

By Theorem 8.4, in a critical clasp the curvature force along $\gamma$ must be balanced by struts to $\tilde{\gamma}$. In particular, almost every point (indeed, since the set of struts is closed, every point) $\gamma(u)$ along the curved arc of $\gamma$ must have a strut to some point $\tilde{\gamma}\left(u^{*}\right)$. Then by symmetry we actually have what we call 2-to-2 contact: there are struts from $\gamma( \pm u)$ to $\tilde{\gamma}\left( \pm u^{*}\right)$. Here the two points $\tilde{\gamma}\left( \pm u^{*}\right)$ must be the intersection of the unit normal circle around $\gamma(u)$ with the $y z$-plane, implying that $u^{*} \in[0,1]$ is uniquely determined for each $u$. We will refer to $\gamma(u)$ and $\tilde{\gamma}\left(u^{*}\right)$ as conjugate points on the $\tau$-clasp. Lemma 9.3 applies to any pair of conjugate points, with $u_{1}=u, u_{2}=u^{*}$ and $x_{i}=x\left(u_{i}\right)$.

Lemma 9.4 Suppose $\gamma$ is a plane curve, symmetric across a line $\ell$ in the plane. Consider the net curvature force of a mirror image pair of infinitesimal arcs of $\gamma$. This acts in the direction of the line $\ell$, with magnitude $2|\mathrm{~d} u|$. Here the function $u$ is defined along $\gamma$ as the cosine of the angle $\psi$ between $\ell$ and the tangent line to $\gamma$.

Proof One infinitesimal arc has net curvature force $\kappa N \mathrm{~d} s=N \mathrm{~d} \psi$. When this is added to the mirror image force, only the component along $\ell$ survives. We get magnitude $2|\sin \psi \mathrm{d} \psi|=2|\mathrm{~d} u|$.

Suppose now we have a symmetric configuration of the clasp where the curved arcs of the two components stay a constant distance 1 apart. By symmetry we get the 2-to-2 strut pattern described above. Assuming the straight ends of each component meet the 
constraint planes perpendicularly, our balance criterion Theorem 8.4 says that strong criticality is equivalent to the statement that the net vertical curvature force exerted by the arcs at $\gamma( \pm u)$ balances that of the conjugate arcs at $\tilde{\gamma}\left( \pm u^{*}\right)$. That is, using Lemma 9.4, for a critical clasp we must have $|\mathrm{d} u|=\left|\mathrm{d} u^{*}\right|$, meaning that either $u-u^{*}$ or $u+u^{*}$ is constant.

If $u-u^{*}$ were constant, by symmetry it would be zero, and our equations would describe a pair of half-ellipses, with horizontal major axis $\sqrt{2}$ and vertical minor axis 1 . On these curves, corresponding points $\gamma(u)$ and $\tilde{\gamma}(u)$ are always at distance 1 from each other, but these are maxima for the distance between components, rather than minima. This configuration has LThi $<1$, and is not LThi-critical: the pairs $\{\gamma(u), \tilde{\gamma}(u)\}$ are not struts.

Instead we must have that $u+u^{*}$ is constant. To find the constant, note that on the $\tau$-clasp, the tip of $\gamma$ (at $u=0$ ) is joined by a strut to the end of $\tilde{\gamma}$ (at $u^{*}=\tau$ ); thus $u+u^{*}=\tau$. This equation holds when $0 \leq u, u^{*} \leq \tau$; to allow for negative values (parametrizing the whole clasp curve) we write

$$
|u|+\left|u^{*}\right|=\tau .
$$

We can now give an explicit description of our critical $\tau$-clasp:

Theorem 9.5 Let $\tau \in[0,1]$, and let $\gamma=\gamma_{\tau}$ be the curve in the $x z$-plane given parametrically for $u \in[-\tau, \tau]$ by

$$
\begin{gathered}
x=x_{\tau}(u):=\frac{u \sqrt{1-(\tau-|u|)^{2}}}{\sqrt{1-u^{2}(\tau-|u|)^{2}}}, \\
z=z_{\tau}(u):=\int \frac{\mathrm{d} z}{\mathrm{~d} x} \mathrm{~d} x=\int \frac{u}{\sqrt{1-u^{2}}} \frac{\mathrm{d} u}{\kappa_{\tau}(u)}, \\
\kappa_{\tau}(u):=\frac{\sqrt{\left(1-u^{2}(\tau-|u|)^{2}\right)^{3}\left(1-(\tau-|u|)^{2}\right)}}{1-(\tau-|u|)^{2}+(\tau-|u|)|u|\left(1-u^{2}\right)}
\end{gathered}
$$

where

and the constant of integration for $z$ is chosen so that

$$
z(0)+z(\tau)=-\sqrt{1-\tau^{2}} .
$$

Then the union of $\gamma$ with its image $\tilde{\gamma}$ under the symmetry group $2 * 2$ described above is a $\tau$-clasp that is strongly critical for link-ropelength. The curvature of $\gamma$ is $\kappa_{\tau}(u)$ above, and the total length of the curved part of $\gamma$ is

$$
\int_{-\tau}^{\tau} \frac{\mathrm{d} u}{\kappa_{\tau}(u) \sqrt{1-u^{2}}}
$$


Proof The proposition follows from the foregoing discussion, after substituting $u^{*}=$ $\tau-|u|$ into the equations of Lemma 9.3, and using Lemma 9.2. To get the constant of integration for $z$, we note that the strut from $\gamma(0)$ to $\tilde{\gamma}(\tau)$ has height given (as in the proof of Lemma 9.3) by

$$
\Delta z=\sqrt{1-x_{\tau}(0)^{2}-x_{\tau}(\tau)^{2}}=\sqrt{1-0-\tau^{2}} .
$$

Although the formulas we have given for $z_{\tau}(u)$ and for arclength both involve hyperelliptic integrals not expressible in closed form, it is straightforward to integrate them numerically; we have plotted our critical configuration of the simple $(\tau=1)$ clasp in Figure 12.

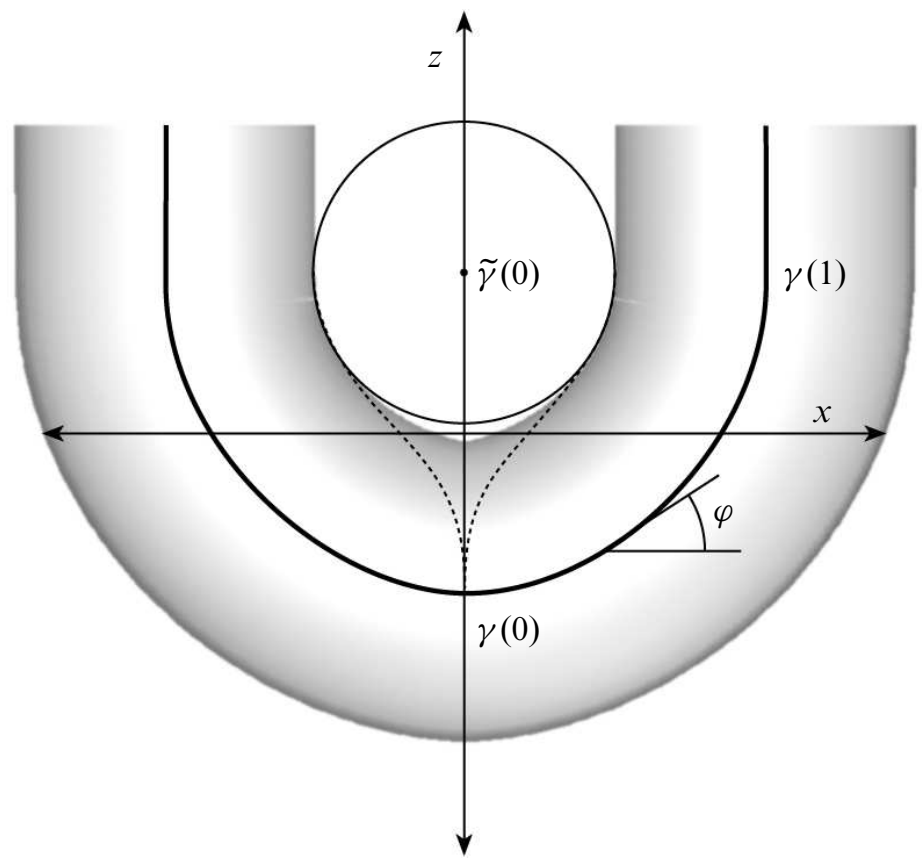

Figure 12: This is an accurate plot of the critical simple clasp $\gamma$ given by Theorem 9.5. Here $u=\sin \varphi$ ranges from -1 to 1 over the curved portion of $\gamma$. The tip $\tilde{\gamma}(0)$ of the other component is shown above $\gamma$ on the $z$-axis, along with the (dotted) circular cross-section of the tube of unit diameter around $\tilde{\gamma}$. The curved dotted lines extending down from the sides of this cross-section are the lines of contact between the shaded tube around $\gamma$ and the front half of the tube around $\tilde{\gamma}$. Symmetric lines of contact extend behind the shaded tube, realizing the 2-to-2 contact pattern we have described. Finally, we see a small gap between the tubes, explored in more detail in Figure 14. 
As we mentioned in the introduction, Starostin has given [34] an independent derivation (using a form of balancing for smooth curves) of these same $\tau$-clasp configurations (as well as the family of stiff clasps we will consider in [2]). Starostin does not prove that these configurations are critical for link-ropelength.

\subsection{The geometry of the tight clasp}

We now examine the curvature and other geometric features of the critical clasps for the Gehring problem that were given in Theorem 9.5. Each component of the critical $\tau$-clasp is a $C^{1}$ join of four analytic pieces: a straight segment, then $\gamma[-\tau, 0]$, then $\gamma[0, \tau]$, and finally another straight segment. Where the curved arcs join the straight segments at $u= \pm \tau$, the curvature $\kappa(u)$ approaches 1 ; at these points, our critical clasp agrees to second order with the naively expected circular arcs.

The maximum curvature $\kappa(0)=1 / \sqrt{1-\tau^{2}}$ occurs at the tip. For $\tau<1$, this is finite, and our $\tau$-clasp is $C^{1,1}$. But for $\tau=1$, the curvature blows up (like $|s|^{-1 / 3}$ ) at the tip. In Figure 13 we plot the curvature $\kappa(u)$ for this simple clasp. The curve is $C^{1,2 / 3}$ (and is also in the Sobolev space $W^{2,3-\varepsilon}$ for all $\varepsilon>0$ ) but has no higher regularity.

In Proposition 6.5, we proved that critical curves for link-ropelength are $C^{1}$. It would be interesting to find out whether all such critical curves are $C^{1,2 / 3}$; perhaps the simple clasp exhibits the worst possible behavior.

In Example 7.5, we saw critical curves constrained by link-thickness which fail to have positive thickness in the ordinary sense of [4] because one component is nonembedded. The simple clasp fails to have positive thickness for a different reason: its curvature is unbounded. In [2] we will consider a family of thickness measures with a variable stiffness parameter $\lambda$. In these measures, a unit-thickness curve has curvature bounded above by $2 / \lambda$. For any nonzero $\lambda$, it follows that the critical simple clasp must be different from the tight clasp here for the Gehring problem, and must instead include an arc of this maximum allowed curvature.

One of the most interesting features of the clasp is the gap between the two components of the clasp. The distance between the tips of $\gamma$ and $\tilde{\gamma}$ is $z(\tau)-z(0)+\sqrt{1-\tau^{2}}$ (written in this way to be independent of the constant of integration for $z$ ). This is an increasing function of $\tau$, close to 1 when $\tau$ is small, but increasing to 1.05639 at $\tau=1$. Thus, in the simple clasp, the gap between the thick tubes around the two components at their tips is almost $6 \%$ of their diameter.

These thick tubes contact each other at the midpoints of the struts. Topologically, the set of struts forms a loop. Their midpoints form a loop in space with four vertical cusps - the line of contact of the two tubes-as seen in Figure 12 and Figure 14. 


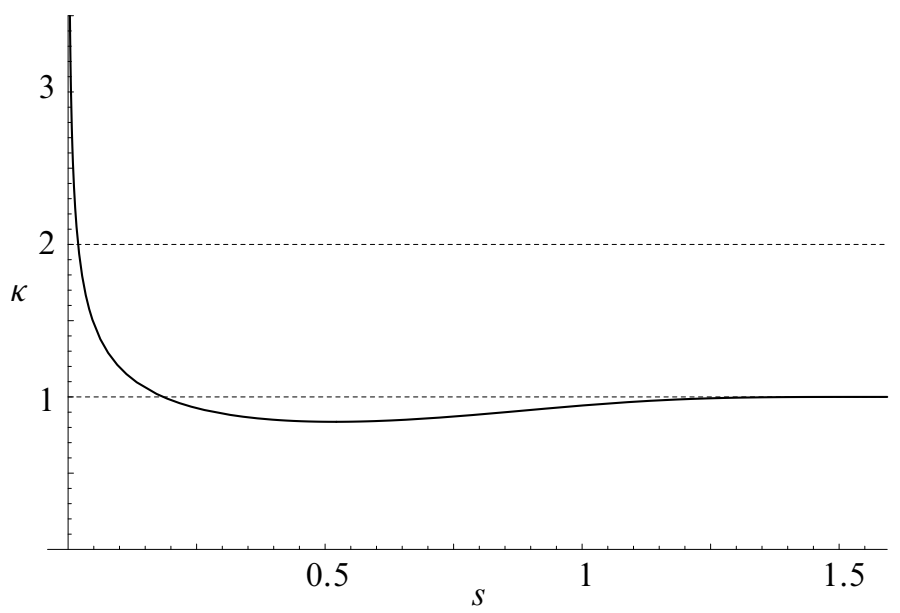

Figure 13: The graph shows the curvature $\kappa$ of the tight 1-clasp as a function of arclength. The curvature blows up at the tip: this curve is only $C^{1,2 / 3}$. The unit-diameter thick tube around the curve forms a cusp near the tip, when the curvature exceeds 2 . From the tip, curvature decreases rapidly to its minimum, and then increases again to the limiting value of $\kappa=1$ at the end. Thus the clasp curve, at its end, agrees to second order with the naively expected unit circle around the tip of the other component, as is suggested in Figure 12. (For $\tau=1$, as illustrated here, the curves agree even to third order.)

Alternatively, we can plot the loop of struts as pairs of arclength coordinates on the two components, as in Figure 15. The solid tubes divide the rest of the ambient space into two regions: one infinite component around the outside of the clasp, and one small chamber sitting in the gap between the tips, shown in Figure 14. To give a sense of scale, the gap chamber has a substantial surface area of about 1.10 , equal to the area of a section of tube of length more than $1 / 3$. However, the chamber is very thin, resulting in a volume of only 0.01425 .

\subsection{Length comparison with the naive clasp}

Earlier, we described the naive circular configuration for the simple clasp. Similarly, in what we call the naive $\tau$-clasp, each component is built from straight segments (normal to the constraint planes) and a unit-radius arc (of angle $2 \arcsin \tau$ and centered at the tip of the other component). As we saw for $\tau=1$, this configuration is not critical: there is no way to balance the forces concentrated on the tips, unlike in Example 7.1 and Example 9.1, which had extra struts. 

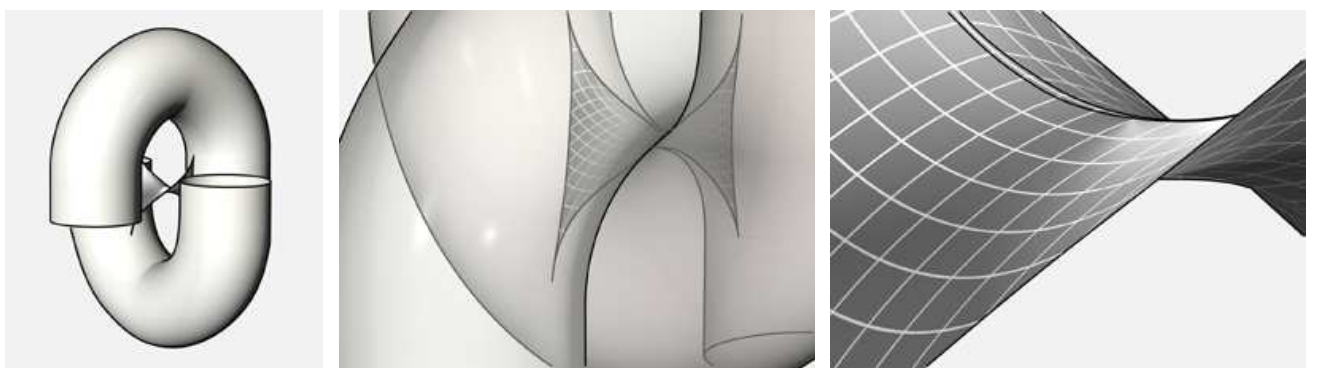

Figure 14: We see three views of the gap chamber between the two tubes in the tight clasp with $\tau=1$. On the left, we see an exploded view with the two tubes and the gap chamber floating between them. In the medium closeup in the center, we see the chamber in place between the (now transparent) tubes. On the right, we see an extreme closeup of the center of the gap chamber. Its height at the center (about 0.05639) is the distance between the tubes at the tips of the clasp. The grid in the center and right pictures is a square grid projected from the $x y$-plane. On the right, we see a tiny ridge running from left to right along the surface of the chamber; this is a cusp formed by the folding of the tube surface that happens when the curvature of the clasp rises above 2 (compare Figure 13). We do not know whether this gap chamber forms in clasps of physical rope; it would be very interesting to find out.

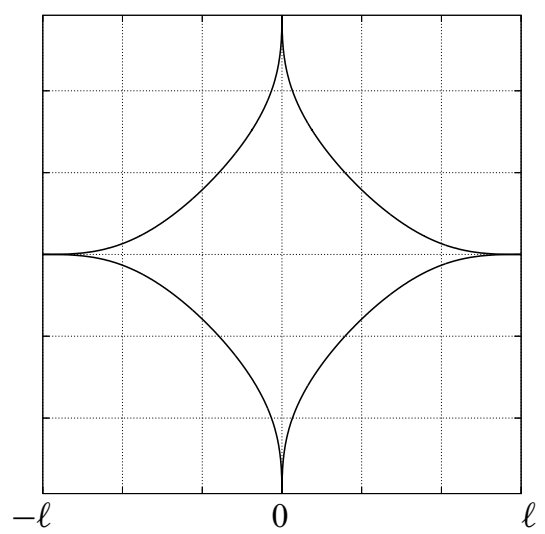

Figure 15: The graph shows the strut set for the tight 1-clasp, where each strut is plotted according to the arclength of its ends on the two components of the clasp (measured from the tip $s=0$ to the shoulders at $s=\ell \approx 1.58944$ ). There is a closed loop of struts, with four cusps at the tips and shoulders of the clasp arcs. We hope this explicit strut set will help in verifying the accuracy of numerically computed strut sets for ropelength minimizers, such as those in [5]. 
Our critical $\tau$-clasps (which we expect are the global minima for length) are indeed slightly shorter than the naive configurations. The total length of a clasp depends, of course, on the position of the bounding planes. Thus to compare the lengths of the naive clasp and our critical clasp in a meaningful way, we introduce the notion of excess length. The infimal possible length of a $\tau$-clasp with no thickness constraint is easily seen to be four times the inradius of the bounding tetrahedron. (In the case $\tau=1$ this is twice the thickness of the bounding slab.) The excess length of any given clasp is the amount by which its length exceeds this value.

For $\tau=1$, the naive clasp has excess length $2 \pi-2$, since two unit semicircles replace two straight segments of unit length. Numerical integration reveals the excess length of our critical 1-clasp to be 4.262897 (accurate to the number of digits shown). It is thus about 0.020288 , or almost half a percent, shorter. In general, the excess length of the naive $\tau$-clasp is $4 \arcsin \tau-2 \tau$, while the excess length of our critical $\tau$-clasp equals the total length of the curved parts minus $2 \tau$ times the inter-tip distance. The maximum percentage savings, about $0.518 \%$, occurs for $\tau \approx \sin \left(80^{\circ}\right)$.

\section{The Borromean rings}

The original Gehring link problem was solved by the Hopf link made from a pair of circles through each other's centers. We have already generalized this to a three component link in one way: the simple chain made from circles and stadium curves of Section 7. But the simple chain is just a connect sum of Hopf links, and so the minimizing configuration shares much of its geometry with the original Gehring solution.

We now construct a proposed minimizer for a more interesting Gehring problem-the Borromean rings (see Figure 16). Among the three prime six-crossing links of three components, the Borromean rings form the one which is Brunnian, meaning that if any one component is removed the remaining components are unlinked. Milnor's $\mu-$ invariant classifies three-component Brunnian link-homotopy types, and the Borromean rings are the first nontrivial example.

In this section, we describe (Theorem 10.2) a critical configuration $B_{0}$ of the Borromean rings, shown in Figure 16 and Figure 17. Numerical simulations with Brakke's Evolver [1] suggest that this configuration $B_{0}$ is in fact the ropelength minimizer for the Borromean rings. We will see below that the curvature of $B_{0}$ stays below 1.534; this means (as we show in [2]) that $B_{0}$ is also a critical point for length when constrained by the ordinary thickness measure of [4] instead of by link-thickness. In [4], we described a similar configuration $B_{2}$ of the Borromean rings, built entirely from arcs of unit 


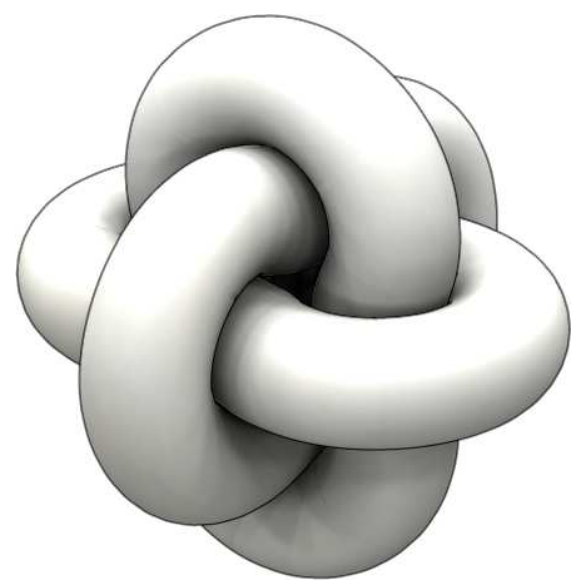

Figure 16: Our critical configuration $B_{0}$ of the Borromean rings, shown with thick tubes of diameter 1 . This configuration is very slightly shorter than the piecewise-circular version in [4]. As in that version, the core curve of this tube has discontinuous curvature, for instance at the "jump point" where the curve switches from convex to concave.

circles. Theorem 6.1 shows that $B_{2}$ is not critical, and we compute that $B_{0}$ is $0.08 \%$ shorter.

\subsection{Symmetry and convexity}

Our configurations $B_{0}$ and $B_{2}$ of the Borromean rings are quite similar, and in particular have the same symmetry and convexity properties, which we now define. The three congruent components lie (respectively) in the three coordinate planes; reflection across any one of these planes is a symmetry of the link preserving each component. A further symmetry, which cyclically permutes the three components, is given by $120^{\circ}$ rotation about the $(1,1,1)$ axis; we write this rotation as

$$
p \mapsto \tilde{p} \mapsto \hat{p} \mapsto p .
$$

These symmetries generate the pyritohedral point group of order 24 in $O(3)$ whose Conway-Thurston orbifold notation $[9 ; 10]$ is $3 * 2$. Algebraically it is isomorphic to $\pm A_{4}$, and in cartesian coordinates it is most naturally seen as the wreath product $\{ \pm 1\}<C_{3}$.

Any symmetric configuration of the Borromean rings is the image under the pyritohedral group of a single embedded arc in the closed positive quadrant of the $x y$-plane, extending from a point $I$ on the $x$-axis to a point $T$ on the $y$-axis, as shown in 

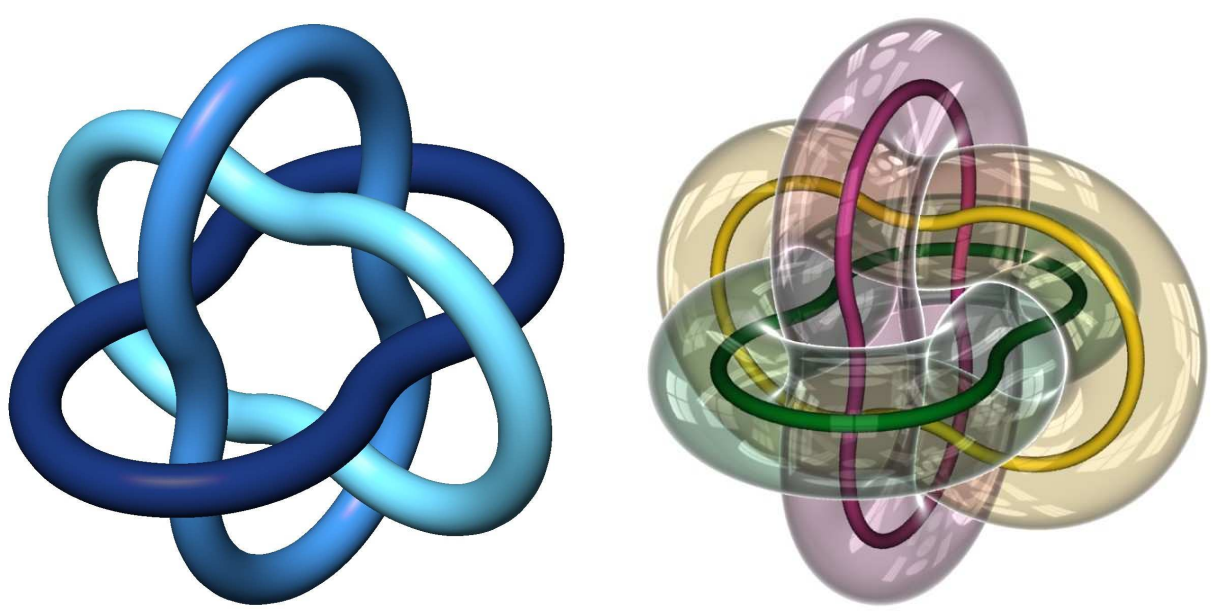

Figure 17: Two further renderings of the critical configuration $B_{0}$ for the Borromean rings reveal more of the structure. The image on the left, showing thin tubes of diameter 0.315 , viewed along an axis of threefold symmetry, has been adopted as the logo of the International Mathematical Union. On the right, in a still from the video [15], we see even thinner tubes inside transparent thick tubes.

Figure 18. Conversely, given any such arc $I T$, its images under $3 * 2$ will form a link isotopic to the Borromean rings, as long as $T$ and $I$ are not at the same distance from the origin. We will assume that $|I|<|T|$ and will call $I$ the intip while $T$ is the tip. To make the link $C^{1}$, the arc $I T$ must be $C^{1}$ and must meet the axes perpendicularly at its endpoints.

The only other points of the link in this quadrant of the $x y$-plane are $\tilde{I}$ and $\widehat{T}$; they will be important in the following discussion.

The arcs $I T$ of interest to us consist of a small concave piece near the intip joined to a large convex piece ending at the tip. That is, there is a jump point $J \in I T$ such that the arc $I J$ is strictly concave, while $J T$ is strictly convex. As in our discussion of the clasp, we will parametrize $I J$ by the angle $\psi$ (less than $\frac{\pi}{2}$ ) that its tangent vector makes to the right of the vertical, or by $v=\sin \psi$. Here $v$ ranges from 0 at $I$ to some value $\sigma$ at $J$, which will be one of the fundamental parameters for the curves we describe.

Along the convex arc $J T$ we can still define $v=\sin \psi$, which now decreases from $\sigma$ through 0 to -1 . But we also use the angle $\varphi=\frac{\pi}{2}+\psi$, the angle above the horizontal made by the tangent vector to $J T$. Since our curve is $C^{1}$, we have $\varphi(T)=0$ and 


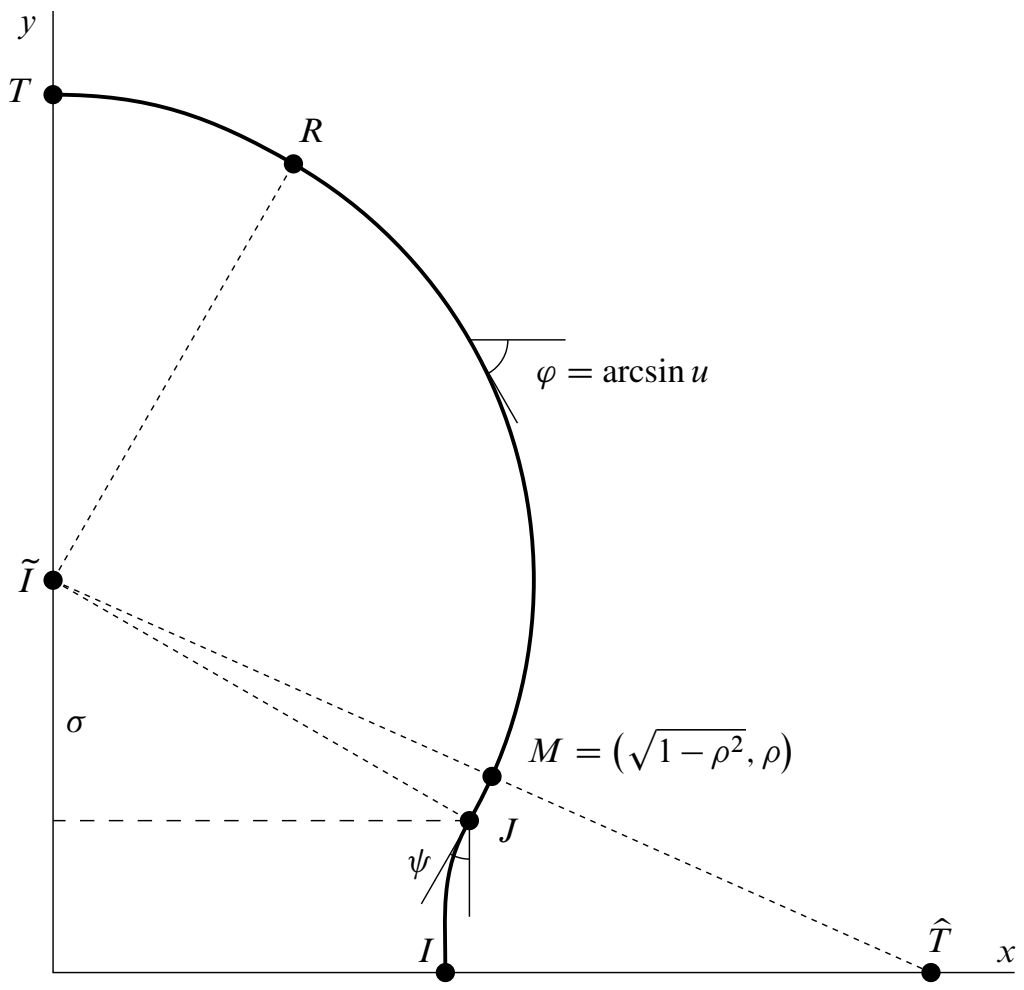

Figure 18: Any configuration $B$ of the Borromean rings with $3 * 2$ symmetry is generated by a planar arc $I T$. We consider arcs where $I J$ is concave and $J T$ is convex. The other points of $B$ in this quadrant are the rotation images $\tilde{I}$ and $\hat{T}$ of $I$ and $T$. In our configurations, there are points $M$ and $R$ such that $J R$ is part of the unit circle around $\widetilde{I}$, and $M$ is the midpoint between $\tilde{I}$ and $\hat{T}$. The four dotted lines are thus struts of length 1 . The height difference from $J$ to $\widetilde{I}$ is $\sigma=\sin \psi(J)$ as delineated by the horizontal dashed line, and the coordinates of $M$ are given in terms of $\rho=\sin \psi(M)=$ $-\cos \varphi(M)$.

$\varphi(J)=\frac{\pi}{2}+\arcsin \sigma$. In the curves we describe, some initial subarc $J R$ of $J T$ is part of the unit circle around $\tilde{I}$; we have $\varphi(R) \leq \frac{\pi}{2}$ so that along $R T$ we can also use the parameter $u=\sin \varphi$.

Finally, to achieve a force balance we will find it necessary that some point $M$ along the circle $J R$ has a strut to $\widehat{T}$ as well as to $\tilde{I}$. This lets us transmit some force from the large convex arc of one component to the smaller concave arc of another, indirectly through the third component. In the $x y$-plane, we find that $M$ is the midpoint of the 
segment $\tilde{I} \hat{T}$, and thus if we set $\rho:=\sin \psi(M) \leq \sigma$ we have

$$
\tilde{I}=(2 \rho, 0), \quad M=\left(\rho, \sqrt{1-\rho^{2}}\right), \quad \hat{T}=\left(0,2 \sqrt{1-\rho^{2}}\right) .
$$

\subsection{The configuration built from circular arcs}

The configuration $B_{2}$ we described in [4] is generated by an arc IT of this form. In $B_{2}$, we have $R=T$, so that the entire convex arc $J T$ is part of the unit circle around $\tilde{I}$. Furthermore, the concave arc $I J$ is also part of a unit circle, centered at $\hat{T}$. This implies that $M=J$ and $\sigma=\rho=: \rho_{2}$. The value $\rho_{2}$ is determined by the fact that $I$ and $\hat{T}$ are at unit distance, meaning $2 \rho_{2}+1=2 \sqrt{1-\rho_{2}^{2}}$. As we computed in [4], the total length of $B_{2}$ is then $6 \pi+24 \arcsin \rho_{2} \approx 29.0263$.

This configuration is not balanced (and thus not critical) for link-ropelength. To balance the curvature forces of the circular arcs, the fans of struts to their centers would have to carry force proportional to arclength. But these struts would then concentrate outward force on the tips and inward force on the intips; there are no further struts to balance these forces. This is like the picture for the naive clasp - all the force is concentrated on the tips. As for the clasp, the tips in the critical configuration will be further apart.

In [2], we introduce a family of thickness measures with variable stiffness. For stiffness 2 (meaning that the curves cannot have osculating circles of diameter less than 2) we will see that $B_{2}$ is balanced and hence critical for ropelength. Because the circular arcs have exactly the maximum allowed curvature, we will see that their curvature force need not be balanced pointwise, but only in total. Outward strut force on their midpoints (the tips and intips) can in a sense be spread out to balance the curvature all along the arc. Because $\rho_{2} \neq 45^{\circ}$, however, there is an imbalance of total curvature forces between the convex and concave arcs. Thus our balancing measure will need an atom of force on the special colinear struts $\{\widetilde{I}, M\}$ and $\{M, \widehat{T}\}$; this transmits force from $\widehat{T}$ through $M$ to $\tilde{I}$.

\subsection{Configurations involving clasp arcs}

To get a balanced configuration $B_{0}$ of the Borromean rings, we have to replace the concave circular arc $I J$ (and part of the convex arc) by a tight clasp arc. Suppose $I J$ is part of a $\tau$-clasp for some $\tau \geq \sigma$. We will now describe a configuration determined by certain values of our three parameters

$$
0 \leq \rho \leq \sigma \leq \tau \leq 1,
$$

a particular curve of the class illustrated in Figure 18. 
First, the arc $I J$ is the piece $v \in[0, \sigma]$ of the $\tau$-clasp, translated out along the $x$-axis until its tip $I$ is at $(2 \rho, 0,0)$. Next, $J M R$ is an arc of the unit circle around $\tilde{I}$, with $v(J)=\sigma, v(M)=\rho$ and $u(R)=\tau$. Note that to get these arcs to match up at $J$, we will need two conditions on our parameters $\rho, \sigma$ and $\tau$. Finally to define the remaining arc $R S T$, consider the image $\tilde{I} \widetilde{J} \tilde{M}$ of $I J M$, rotated into the $y z$-plane. Then $R S T$ is conjugate to $\tilde{I} \widetilde{J} \tilde{M}$ in the sense of Lemma 9.3: it is the intersection of the unit-radius tube around $\tilde{I} \widetilde{J} \tilde{M}$ with the $x y$-plane, with $S$ defined to be the point conjugate to $\widetilde{J}$. Figure 19 shows the arc $I T$ and its two rotated images, that is, the part of $B_{0}$ lying in the nonnegative orthant in space.

Lemma 10.1 For any fixed $\tau$, suppose the parameters $0 \leq \rho \leq \sigma \leq \tau$ satisfy the two equations

$$
\begin{aligned}
& 0=2 \rho-\sqrt{1-\sigma^{2}}+\int_{u=0}^{u=\sigma} \frac{u \mathrm{~d} u}{\kappa_{\tau}(u) \sqrt{1-u^{2}}}, \\
& 0=1-(2 \rho-\sigma)^{2}-\frac{1-\sigma^{2}}{1-\sigma^{2}(\tau-\sigma)^{2}},
\end{aligned}
$$

where $\kappa_{\tau}$ is the curvature of the clasp from Theorem 9.5. Then there is a $C^{1}$ and piecewise analytic arc IJMRST as described in the last paragraph. Its images under the symmetry group $3 * 2$ form a configuration $B(\rho, \sigma, \tau)$ of the Borromean rings with link-thickness LThi $=1$.

Proof As a point on the unit circle $J R$ around $\tilde{I}$, the jump point $J$ has coordinates

$$
\left(\sqrt{1-\sigma^{2}}, 2 \rho-\sigma, 0\right) .
$$

As a point on the $\tau$-clasp $I J$, its coordinates are

$$
\left(2 \rho+\int_{0}^{\sigma} \frac{u \mathrm{~d} u}{\kappa_{\tau}(u) \sqrt{1-u^{2}}}, x_{\tau}(\sigma), 0\right)
$$

Equating these, using

$$
x_{\tau}^{2}(\sigma)=1-\frac{\left(1-\sigma^{2}\right)}{1-\sigma^{2}(\tau-\sigma)^{2}}
$$

from Theorem 9.5, gives (10-1) and (10-2).

If these equations are satisfied, then the position of $J$ is well-defined, and $I J R$ is a $C^{1}$ arc, meeting the $x$-axis perpendicularly. The arc $R S T$ is the conjugate of $I J M$ and thus is $C^{1}$ by Lemma 9.3. At $T$, the same lemma shows it meets the $y$-axis perpendicularly. At $R$, the $u=\tau$ base of the $\tau$-clasp agrees even to second order with the unit circle. 


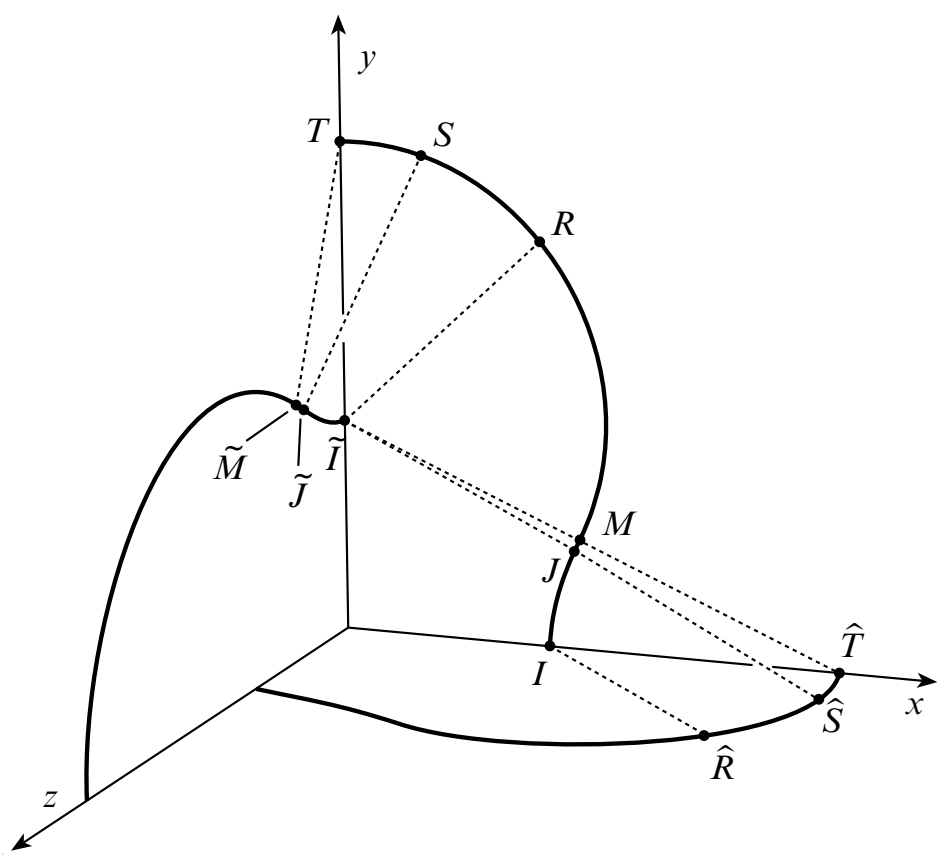

Figure 19: One octant of the critical Borromean rings $B_{0}$ consists of three rotated images of an arc IJMRT of the type shown in Figure 18. The dotted lines are struts of length 1 connecting the labeled points. We now describe all other struts to $I T$ in this octant. Of course, all along the circular $\operatorname{arc} J M R$ there are struts to its center $\widetilde{I}$. Also, between the marked struts are several one-parameter families of struts, joining two arcs. The first family joins the conjugate clasp arcs $R S$ and $\widetilde{I} \widetilde{J}$; a second family connects $S T$ to the circular arc $\tilde{J} \tilde{M}$. The other families are rotated images of these, connecting $J M$ to $\hat{S} \hat{T}$, and $I J$ to $\hat{R} \hat{S}$. The struts $\{\widehat{T}, M\}$ and $\{M, \widetilde{I}\}$ are colinear. To balance $I T$, it is important to consider also the mirror-image struts across the $x y$-plane. This figure is an accurate drawing of $B_{0}$, except that we have exaggerated the separation between $M$ and $J$ : their actual distance is smaller than the width of the lines used in the picture.

In this configuration, all the struts shown in Figure 19 have length 1. If the linkthickness were less than 1 , there would need to be some shorter strut in this positive octant. But that strut would be governed by Lemma 9.3, and (rotating to assume one endpoint is on $I T$ ) its projection to the $x y$-plane would be normal to the arc $I T$; the figure makes it clear that no such strut exists. 


\subsection{The balanced configuration}

Finally, we wish to find the third condition on our parameters $\rho, \sigma$ and $\tau$, which will ensure that $B(\rho, \sigma, \tau)$ satisfies the balance criterion of Corollary 6.3.

For most of the struts, it is immediately clear what stress they need to have in a balancing measure $\mu$ : The struts from $I J$ to $\widehat{R} \widehat{S}$, and those from $M R$ to $\widetilde{I}$ and from $R T$ to $\widetilde{I} \tilde{M}$ must be stressed exactly enough to balance the curvature force of $I J$ and of $M R T$. The conjugate clasp arcs $I J$ and $R S$ exactly balance each other's curvature forces in this way.

The situation along the short circular arc $J M$ is more complicated. The struts inward to $\widetilde{I}$ need to balance not only the curvature force of $J M$ itself, but also the force acting inward on $J M$ from the struts from $\widehat{S} \widehat{T}$. Remember that the measure needed on these last struts is determined by the curvature of $\widehat{S} \widehat{T}$; this in turn determines the measure needed on the inward struts from $J M$. We will write this down explicitly below. The final condition on our parameters then comes from a balance of forces at $\widetilde{I}$, where a whole family of struts converges.

Note that this configuration $B_{0}$ of the Borromean rings is the first known example of a ropelength-critical configuration in which this sort of transmitted force appears. Struts impinge on the arc $J M$ from the direction opposite its own curvature, and transmit their force through that arc. Without this force transmitted through the (very) short arc $J M$, the relatively long convex piece $\widehat{R} \widehat{T}$ would exert too much inward force on the relatively short concave piece $I J$. Instead, some of this inward force, when transmitted through $J M$, becomes force outward on the concave piece $\tilde{I} \widetilde{J}$. This transmitted force plays the same role in balancing $B_{0}$ that the atomic force from $\widehat{T}$ through $M$ to $\tilde{I}$ played in balancing $B_{2}$ for the stiff problem. But here our strut measure is absolutely continuous, with no atoms.

To write down the final balancing condition at $\tilde{I}$, we begin with an application of Lemma 9.4: the total curvature force of $J M R$ and its mirror image across the $y z$-plane acts on $\tilde{I}$ downward in the $y$-direction, with magnitude

$$
2(u(J)-u(R))=2\left(\sqrt{1-\sigma^{2}}-\tau\right) .
$$

But the struts from $J M$ carry extra transmitted force. To determine this, consider the curvature force of an infinitesimal arc of $\hat{S} \hat{T}$ and its mirror image across the $x y$-plane. Parametrizing them as usual by $u$, Lemma 9.4 tells us the net force, exerted in the negative $x$ direction, is $2 \mathrm{~d} u$. This horizontal force is exerted on an infinitesimal piece of $J M$ and its mirror image across the $x z$-plane. If we parametrize $J M$ by $v=\sin \psi$, then remembering that the force on this arc acts perpendicular to the arc, we see that 
if its horizontal component is $\mathrm{d} u$, then its vertical component is $v \mathrm{~d} u / \sqrt{1-v^{2}}$. This force gets transmitted through to $\widetilde{I}$. Because of the symmetry across the $y z$-plane, of course only the vertical component matters in the end. But this symmetry also doubles that vertical force. (Four copies of the $\operatorname{arc} \hat{S} \hat{T}$ act on $\tilde{I}$ : the original, and reflections across the $x y$ - and $y z$-planes.) The resultant total transmitted force on $\widetilde{I}$ is upward with magnitude

$$
2 \int_{u=0}^{\tau-\sigma} \frac{2 v}{\sqrt{1-v^{2}}} \mathrm{~d} u
$$

Here the upper limit of integration is $u(S)=\tau-v(J)$ because $J$ and $S$ are conjugate points on the $\tau$-clasp. To make this integral explicit, we need to give the relation between $u$ and $v$; this comes from Lemma 9.3. Along $J M$ we have $y$-coordinate $2 \rho-v$, so the lemma gives

$$
u^{2}=u(v)^{2}:=\frac{1-(2 \rho-v)^{2} / v^{2}}{1-(2 \rho-v)^{2}} .
$$

If one wanted, this could be solved to give $v$ as the root of a quartic equation in $\rho$ and $u$. Note that $u=0$ at $v=\rho$, as we expect for $T$ and $M$. Plugging in $u=\tau-\sigma$ and $v=\sigma$ (at $S$ and $J$ ) reproduces (10-2).

Summarizing, we can write the force-balancing condition at $\widetilde{I}$ as

$$
0=\tau-\sqrt{1-\sigma^{2}}+\int_{v=\rho}^{v=\sigma} \frac{2 v}{\sqrt{1-v^{2}}} \frac{\mathrm{d} u(v)}{\mathrm{d} v} \mathrm{~d} v
$$

and so we have proved the following theorem.

Theorem 10.2 Suppose $\rho=\rho_{0}, \sigma=\sigma_{0}$ and $\tau=\tau_{0}$ satisfy the three equations (10-1), (10-2) and (10-3). Then the configuration $B_{0}=B\left(\rho_{0}, \sigma_{0}, \tau_{0}\right)$ of the Borromean rings, constructed as in Lemma 10.1, is strongly critical for link-ropelength.

It is easy to solve (10-1) for $\rho$, or (10-2) for $\rho$ or $\tau$, or (10-3) for $\tau$, thereby eliminating one of our three variables. Then we are left with two nonlinear integral equations in the other two variables. While we have not proved formally that a solution to this system exists, we have solved it numerically to high precision, both in Mathematica and using MINPACK [26] and QUADPACK [30]. We obtain

$$
\rho_{0} \approx 0.4074218, \quad \sigma_{0} \approx 0.4177486, \quad \tau_{0} \approx 0.7561107,
$$

where again we follow the standard convention that the error is less than \pm 1 in the last digit shown. There is nothing delicate about this solution, since our expressions vanish to first order at this point. Numerically it is also clear that this solution is unique. 
Using these constants, we compute the length of our critical Borromean rings $B_{0}$ as 29.0030. By comparison, the length of the piecewise circular Borromean rings $B_{2}$ was 29.0263. Thus our critical configuration $B_{0}$ beats the naive circular configuration $B_{2}$ by slightly less than one-tenth of one percent. For comparison, the best lower bound known so far [4] for the length of the Borromean rings is $6 \pi$.

Figure 20 shows an arclength plot of the struts in the Borromean rings. In Figure 21 we plot the curvature of the critical Borromean rings $B_{0}$ as a function of arclength. Note that it is discontinuous only at $J$ and $S$. Each component in $B_{0}$ is built of 14 analytic pieces, joined in a $C^{1,1}$ fashion at the symmetric images of the points $I, J, R$ and $S$. The maximum curvature (at the intips $I$ ) is $\left(1-\tau_{0}^{2}\right)^{-1 / 2} \approx 1.528$. Therefore $B_{0}$ is also ropelength critical for the standard ropelength functional of [4], as we will show in [2]. It is also critical for all the stiff ropelength functionals where the lower bound $\lambda$ on the diameter of curvature is less than $2 \sqrt{1-\tau_{0}^{2}} \approx 1.3$.

We note that Starostin has described [34] a configuration $B_{S}$ of the Borromean rings with ropelength intermediate between that of our $B_{2}$ and $B_{0}$; his configuration replaces the arcs $I J$ and $R T$ of $B_{2}$ by clasp arcs, but does not incorporate the other features of $B_{0}$. While $B_{S}$ can be balanced almost everywhere and Starostin appears to assume that it is a critical configuration, in fact it is not balanced at the intips since it does not satisfy the equivalent of (10-3). Thus by Corollary $6.3, B_{S}$ is not critical.

\section{Open problems and further directions}

Our work in this paper has been motivated by a simple principle: that the ideas of rigidity theory for finite frameworks of bars and struts can be extended to handle mechanisms built from continuous curves of constraints and contacts. In the simple case of links critical for link-ropelength, this method has already yielded some strong results, such as our $C^{1}$-regularity theorem, as well as some surprises like the tight clasp and the critical Borromean rings. Furthermore we expect that these methods in general, and our Kuhn-Tucker Theorem 5.4 in particular, will prove to be useful tools, with applications to a number of outstanding problems in the geometry and topology of curves and surfaces.

We have mentioned our forthcoming extension of these results [2] to the classical ropelength problem, where the presence of curvature constraints and self-contacts of the tube around individual components makes the situation considerably more challenging. Our theory of generalized links and obstacles should also be applicable to the study of packing problems for tubes and surfaces, as when thick rope is packed into a box [18] (a problem of some interest in molecular biology [21;22]), or when the gray 


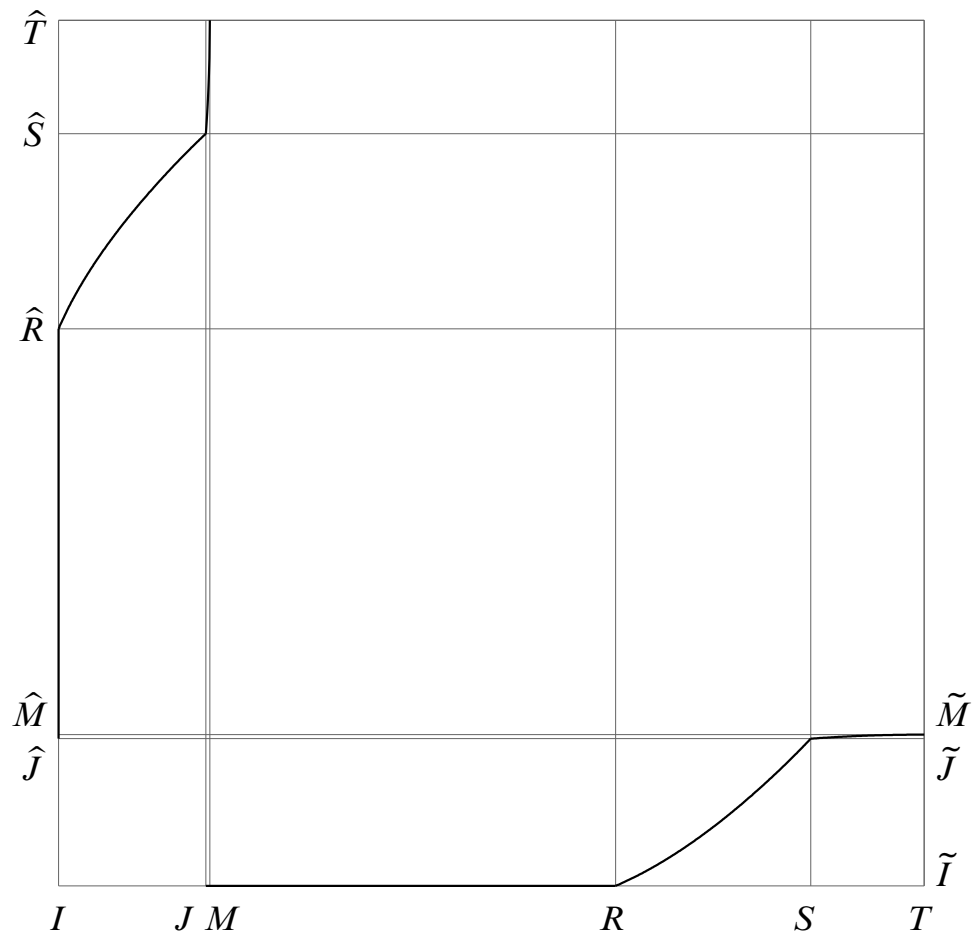

Figure 20: This picture shows a portion of the strut set of our Borromean rings, plotted as pairs of arclength coordinates along components of the link. The horizontal axis represents arclength along one quadrant of the horizontal component, from $I$ to $T$. On the vertical axis, we plot arclength along quadrants of the other two components simultaneously. (This plot accurately depicts the small arclength between $M$ and $J$, in contrast to Figure 19 where this distance is exaggerated.) The horizontal segment at the bottom shows the struts from the circular arc $J M R$ to $\tilde{I}$; it joins to arcs representing the families of struts from $R S$ to $\tilde{I} \widetilde{J}$ and from $S T$ to $\widetilde{J} \tilde{M}$. Symmetrically, the struts to the third component are shown at the upper left: a vertical segment for the circle $\widehat{J} \hat{R}$ around $I$, and arcs for the struts from $I J M$ to $\widehat{R} \hat{S} \widehat{T}$. Remembering that this square plot should be reflected across all of its sides to show the complete strut set, we can easily read off the number of struts coming in to any point on the curve: two along $I J$ and $R S T$, three along $J M$ and one along $M R$.

matter of the brain is folded and pressed against the skull. We should also mention that while we have only considered minimizing length in this paper, our framework should work equally well for other objective functionals, such as a general theory of elastic rods with self-contact. 


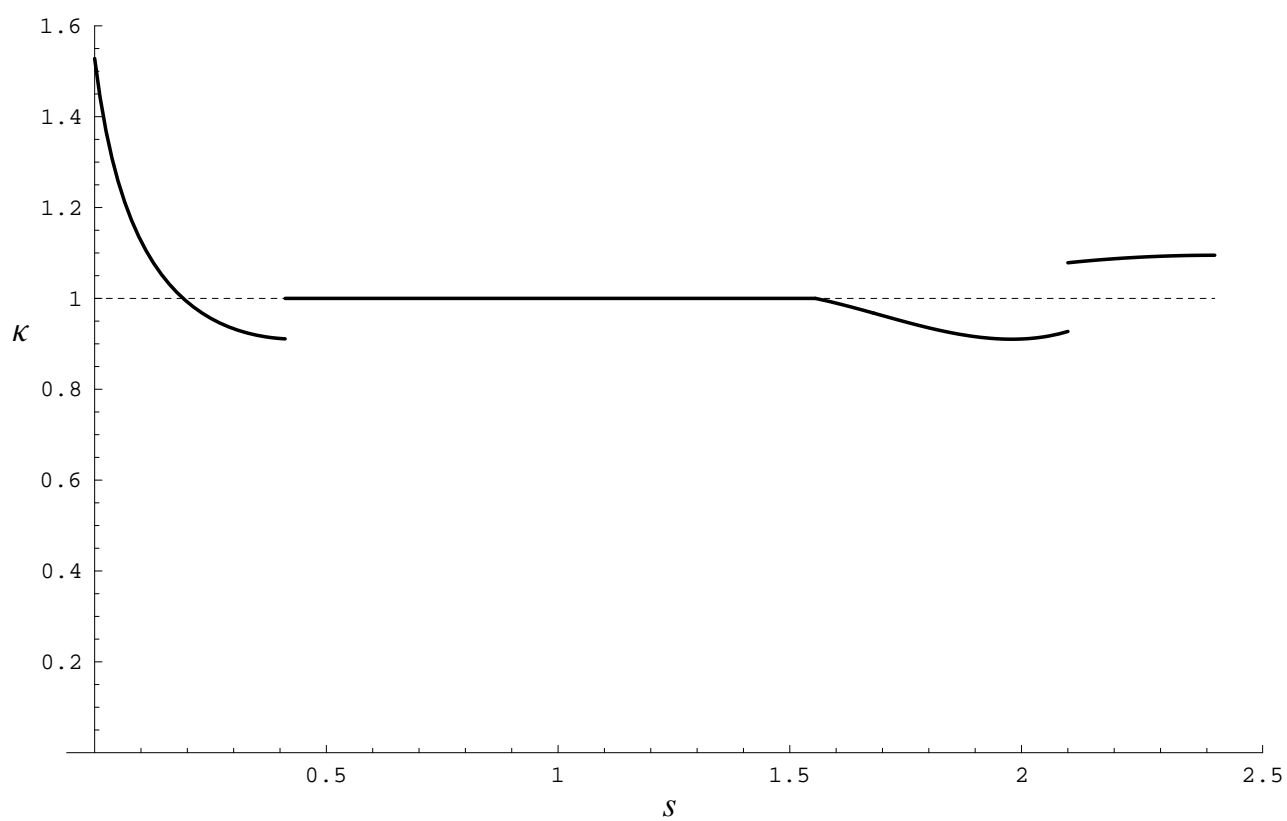

Figure 21: The curvature $\kappa$ of the Borromean rings $B_{0}$, plotted as a function of arclength $s$ along one quarter of one component of this critical configuration. The curvature has its maximum (about 1.533) at the intip $I$, at $s=0$ in this plot, and then smoothly drops off to below 1. (This first part could have been plotted negatively, since this is the concave piece of $B_{0}$, but we have chosen to show the unsigned $\kappa$ of a space curve.) After a jump at $J$, we have $\kappa \equiv 1$ along the circular arc $J M R$. Along the clasp arc $R S$, the curvature drops smoothly from 1 and then rises slightly again, before jumping up above 1 at $S$ and then increasing to a local maximum at $T$.

A finite-dimensional duality theorem akin to our Kuhn-Tucker theorem is one key step in the proof of the Unfolding Theorem of Connelly, Demaine and Rote [8]: Any embedded, nonconvex planar polygon admits a motion that preserves all edge lengths and strictly increases the distance between any two points on the polygon not already joined by a straight line of polygon edges.

Our theory allows us to complete part of the proof of the (conjectured) generalization to smooth plane curves. Whether our methods can be made strong enough to overcome the formidable difficulties involved in proving a smooth unfolding theorem remains to be seen.

There are several specific open questions suggested by our work above. 
Question What is the regularity of a critical curve for link-ropelength? Such curves are at worst $C^{1}$ and at best $C^{1,2 / 3}$.

While we have demonstrated critical configurations of the tight clasps and Borromean rings, we have not attempted to prove that these configurations are minimal.

Question Are our tight clasps and Borromean rings length-minimal in their linkhomotopy types?

The Euclidean-cone methods of [4] seem to hold out some hope for reducing the clasp problem to the case where both curves are planar, but we have not investigated this line of attack.

Acknowledgments We gratefully acknowledge many helpful conversations with our colleagues, including Ted Ashton, Bob Connelly, Erik Demaine, Elizabeth Denne, Oguz Durumeric, Oscar Gonzalez, Xiao-Song Lin, Marvin Ortel and Heiko von der Mosel. In addition, we would like to thank John Maddocks and the Bernoulli Center at EPFL for hosting some of us during some of the summer of 2003, Eric Rawdon and Michael Piatek for sharing their TOROS-minimized link data, and Matt Hoffman for helpful technical advice on the (extraordinary) Electric Image rendering system. Some of the other figures in the paper were prepared with Geomview, avn and RenderMan. This work was partially supported by the NSF through grants DMS-99-02397 (to Cantarella), DMS-02-04826 (to Cantarella and Fu), DMS-00-76085 (to Kusner) and DMS-00-71520 (to Sullivan).

\section{References}

[1] KA Brakke, The surface evolver, Experiment. Math. 1 (1992) 141-165 MR1203871

[2] J Cantarella, J H G Fu, R B Kusner, J M Sullivan, N C Wrinkle, Ropelength criticality, in preparation

[3] J Cantarella, R B Kusner, J M Sullivan, Tight knot values deviate from linear relations, Nature 392 (1998) 237-238

[4] J Cantarella, R B Kusner, J M Sullivan, On the minimum ropelength of knots and links, Invent. Math. 150 (2002) 257-286 MR1933586

[5] M Carlen, B Laurie, J H Maddocks, J Smutny, Biarcs, global radius of curvature, and the computation of ideal knot shapes, from: "Physical and numerical models in knot theory", Ser. Knots Everything 36, World Sci. Publ., Singapore (2005) 75-108 MR2197935 
[6] F H Clarke, Generalized gradients and applications, Trans. Amer. Math. Soc. 205 (1975) 247-262 MR0367131

[7] J Clunie, W K Hayman (editors), Proceedings of the Symposium of Complex Analysis, Held at the University of Kent, Canterbury, 1973, London Mathematical Society Lecture Note Series 12, Cambridge University Press, London (1974) MR0385074

[8] R Connelly, E D Demaine, G Rote, Straightening polygonal arcs and convexifying polygonal cycles, U.S.-Hungarian Workshops on Discrete Geometry and Convexity (Budapest, 1999/Auburn, AL, 2000), Discrete Comput. Geom. 30 (2003) 205-239 MR2007962

[9] J H Conway, O Delgado Friedrichs, DH Huson, WP Thurston, On threedimensional space groups, Beiträge Algebra Geom. 42 (2001) 475-507 MR1865535

[10] J H Conway, D A Smith, On quaternions and octonions: their geometry, arithmetic, and symmetry, A K Peters Ltd., Natick, MA (2003) MR1957212

[11] M Edelstein, B Schwarz, On the length of linked curves, Israel J. Math. 23 (1976) 94-95 MR0397558

[12] M E Gage, A proof of Gehring's linked spheres conjecture, Duke Math. J. 47 (1980) 615-620 MR587169

[13] M E Gage, On Gehring's linked sphere problem, Amer. J. Math. 103 (1981) 437-443 MR618320

[14] O Gonzalez, J H Maddocks, F Schuricht, H von der Mosel, Global curvature and self-contact of nonlinearly elastic curves and rods, Calc. Var. Partial Differential Equations 14 (2002) 29-68 MR1883599

[15] C Gunn, J M Sullivan, The Borromean rings: a new logo for the IMU, Five-minute video available at http://torus.math.uiuc.edu/jms/Videos/imu/

[16] N Habegger, X-S Lin, The classification of links up to link-homotopy, J. Amer. Math. Soc. 3 (1990) 389-419 MR1026062

[17] H W Kuhn, A W Tucker, Nonlinear programming, from: "Proceedings of the Second Berkeley Symposium on Mathematical Statistics and Probability, 1950”, University of California Press, Berkeley and Los Angeles (1951) 481-492 MR0047303

[18] R Kusner, On thickness and packing density for knots and links, from: "Physical knots: knotting, linking, and folding geometric objects in $\mathbb{R}^{3}$ (Las Vegas, NV, 2001)", Contemp. Math. 304, Amer. Math. Soc., Providence, RI (2002) 175-180 MR1953339

[19] D G Luenberger, Optimization by vector space methods, John Wiley \& Sons, New York (1969) MR0238472

[20] O L Mangasarian, S Fromovitz, The Fritz John necessary optimality conditions in the presence of equality and inequality constraints, J. Math. Anal. Appl. 17 (1967) 37-47 MR0207448 
[21] A Maritan, C Micheletti, J Banavar, F Seno, Protein structures and optimal folding from a geometric variational principle, Physical Review Letters 82 (1999) 3372-3375

[22] A Maritan, C Micheletti, A Trovato, J Banavar, Optimal shapes of compact strings, Nature 406 (2000) 287-288

[23] K C Millett, E J Rawdon, Energy, ropelength, and other physical aspects of equilateral knots, J. Comput. Phys. 186 (2003) 426-456 MR1973197

[24] J W Milnor, On the total curvature of knots, Ann. of Math. (2) 52 (1950) 248-257 MR0037509

[25] J Milnor, Link groups, Ann. of Math. (2) 59 (1954) 177-195 MR0071020

[26] J Moré, B Garbow, K Hillstrom, MINPACK http://www.netlib.org/minpack/

[27] A Nabutovsky, Non-recursive functions, knots "with thick ropes", and self-clenching “thick” hyperspheres, Comm. Pure Appl. Math. 48 (1995) 381-428 MR1324407

[28] R Osserman, Some remarks on the isoperimetric inequality and a problem of Gehring, J. Analyse Math. 30 (1976) 404-410 MR0445408

[29] P Pieranski, S Przybyl, A Stasiak, Gordian unknots arXiv:physics/0103080

[30] R Piessens, E de Doncker, CW Ueberhuber, DK Kahaner, QUADPACK http://www.netlib.org/quadpack/

[31] B Roth, W Whiteley, Tensegrity frameworks, Trans. Amer. Math. Soc. 265 (1981) 419-446 MR610958

[32] H L Royden, Real analysis, The Macmillan Co., New York (1963) MR0151555

[33] F Schuricht, H von der Mosel, Characterization of ideal knots, Calc. Var. Partial Differential Equations 19 (2004) 281-305 MR2033143

[34] E L Starostin, A constructive approach to modelling the tight shapes of some linked structures, Forma 18 (2003) 263-293 MR2040086

[35] J M Sullivan, Approximating ropelength by energy functions, from: "Physical knots: knotting, linking, and folding geometric objects in $\mathbb{R}^{3}$ (Las Vegas, NV, 2001)", Contemp. Math. 304, Amer. Math. Soc., Providence, RI (2002) 181-186 MR1953340

[36] J M Sullivan, Curves of finite total curvature, from: "Discrete Differential Geometry", Birkhäuser (to appear in 2007) arXiv:math.GT/0606007

[37] J M Sullivan, N C Wrinkle, Some ropelength-critical clasps, from: "Physical and numerical models in knot theory", Ser. Knots Everything 36, World Sci. Publ., Singapore (2005) 565-580 MR2197958

JC, JHGF: Department of Mathematics, University of Georgia

Athens, GA 30602, USA

Department of Mathematics, University of Massachusetts

Amherst, MA 01003, USA 
Institut für Mathematik, Technische Universität Berlin

DE-10623 Berlin, Germany

Department of Mathematics, Northeastern Illinois University

Chicago, IL 60625, USA

cantarel@math.uga.edu, fu@math.uga.edu, kusner@math.umass.edu, sullivan@math.tu-berlin.de, N-Wrinkle@neiu.edu

Proposed: Yasha Eliashberg

Received: 16 May 2005

Seconded: Joan Birman, Tobias Colding

Revised: 10 October 2006 